\title{
Mathematical General Relativity
}

\author{
Alan A. Coley
}

August 9, 2018

\begin{abstract}
We present a number of open problems within general relativity. After a brief introduction to some technical mathematical issues and the famous singularity theorems, we discuss the cosmic censorship hypothesis and the Penrose inequality, the uniqueness of black hole solutions and the stability of Kerr spacetime and the final state conjecture, critical phenomena and the Einstein-Yang-Mills equations, and a number of other problems in classical general relativity. We then broaden the scope and discuss some mathematical problems motivated by quantum gravity, including AdS/CFT correspondence and problems in higher dimensions and, in particular, the instability of anti-de Sitter spacetime, and in cosmology, including the cosmological constant problem and dark energy, the stability of de Sitter spacetime and cosmological singularities and spikes. Finally, we briefly discuss some problems in numerical relativity and relativistic astrophysics.
\end{abstract}

Alan Coley: Department of Mathematics and Statistics, Dalhousie University, Halifax, Nova Scotia, B3H 4R2, Canada [aac@mathstat.dal.ca]. 


\section{Introduction}

We discuss a number of open problems within classical (mathematical) general relativity (GR). There have been a number of unsolved problems put forward previously. 120 open questions were presented by Yau in 1, mainly in differential (and primarily Riemannian) geometry, but they include five problems in GR: problems Y115, 116 and 119 therein, and problem 114 on cosmic censorship and problem 120 on defining the total angular momentum. These problems were also listed among the fourteen unsolved problems in GR by Penrose (RP12 and 10) in [1, which were mostly formal questions about null infinity, conformal properties and conservation laws, needed for the fundamental problems and conjectures that subsequently followed. From the assumption of cosmic censorship [2] and some reasonable physical properties, a sequence of inequalities can then be derived (e.g., the Penrose inequality RP13 [3]), the validity of which can be interpreted as giving support for cosmic censorship. There were a number of other problems presented, including (as yet unsolved) problems on black holes (e.g., RP14). In addition, many open problems in the vacuum case can be generalized to problems which include matter.

There was also a number of technical problems in mathematical GR presented by Bartnik [4. Indeed, by this time GR had matured sufficiently for mathematical arguments to have replaced previous heuristic arguments and for rigorous problems to be posed. Problem RB32 is the so-called "Bartnik splitting conjecture": Let $M$ be a "cosmological spacetime" satisfying the timelike convergence condition: then either $M$ is timelike geodesically incomplete or $M$ splits as $R \times M^{3}$ isometrically and thus is static (see also Y115 in [1). In the case of a four dimensional (4D) vacuum globally hyperbolic, spatially compact spacetime, if $\mathrm{M}$ splits it is necessarily flat and covered by $R \times T^{3}$, and thus for a non-vacuum "cosmological spacetime" the conjecture asserts that the spacetime either is singular or splits. The basic Lorentzian splitting conjecture RB32 was resolved in [5], and can be regarded as a singularity theorem since a spacetime is timelike geodesically incomplete except in the unphysical cases in which the spacetime splits. The current status of the conjecture by Bartnik has been further reviewed in 6]. In addition, problem RB43 [4] concerned the question of finding an exact 2-body solution of the Einstein field equations and the stability of a system of two orbiting bodies in GR (see also [7]). This is currently important for the study of the merger of two compact objects in which gravitational radiation is copiously produced, especially in light of recent LIGO observations [8. This will be briefly discussed at the end of this paper. 
More recently a (selected) discussion of advances in the mathematical understanding of Einstein's GR was provided by Chrusciel, Galloway and Pollack (CGP) in 9], in which a list of 20 open problems was presented (in Appendix A therein). These problems are often technical and many are specific sub-problems of those discussed in the main text here (where more recent progress is also described) and include initial-boundary-value-problems such as, for example, to find a well posed initial boundary value problem for the vacuum Einstein equations that is well suited for numerical treatment (CGP7) and problems regarding initial data (CGP16 \& 19). In addition, their problem (CGP11) asks whether the existence of Cauchy horizons, not necessarily compact or analytic, implies the existence of local isometries. Another concise survey on the Cauchy problem was given in [10, a number of problems in mathematical GR were discussed in [1], and a survey on strong cosmic censorship is anticipated soon by Dafermos and Luk. 


\section{Open problems in General Relativity}

There are many problems concerning the general mathematical properties of solutions of Einstein's field equations of GR. A sufficiently differentiable 4D Lorentz manifold is considered. The causal structure of the manifold is determined by the Lorentzian metric, which satisfies the Einstein field equations. These equations form a hyperbolic system of quasi-linear partial differential equations which are generally coupled to additional partial differential equations governing the matter in the spacetime [12. First the vacuum case is investigated (with a Ricci flat metric). It is important to study the Cauchy problem; here the unknown variables in the vacuum constraint equations, which consist of a spatial metric and a symmetric 3-tensor belonging to a Riemannian 3D submanifold (and any appropriate initial data for the matter fields), are taken as the initial data for the vacuum evolution field equations.

The Einstein field equations are covariant (that is, invariant under an arbitrary change of coordinates), which further complicates the their formulution [13. The resulting vacuum field equations are not, in the normal sense, hyperbolic. However, in so-called harmonic coordinates the vacuum equations $d o$ form a quasi-linear hyperbolic system and consequently the Cauchy problem is well posed and the usual local existence results follow [16. In addition, the constraints and gauge conditions are are preserved by the evolution equations. In particular, the global regularity for small, smooth initial data (with suitable asymptotic decay) in 3D for a coupled Klein-Gordon-Wave system (which is a simple analogue of the Klein-Gordon system in GR) was investigated in [14. Results for the field equations of GR coupled to a variety of different material fields are also known; these matter fields include not only perfect fluids and gases satisfying kinetic theory, but also scalar fields, Maxwell fields and YangMills fields, and even exotic matter motivated by string theory [15], and various combinations thereof. Perfect fluid results generally only apply when the energy density of the fluid is bounded (uniformly) [12].

Local existence: The standard theorem states that given infinitely differentiable $\left(C^{\infty}\right)$ initial data for the vacuum Einstein field equations there is, defined at least for a finite time period, a smooth solution of the equations which corresponds to this initial data 17. The local existence of solutions of the field equations in GR is reasonably well understood. The basic global uniqueness theorem then guarantees the uniqueness of the maximal development [16] of any such initial Cauchy data (up to diffeomorphisms that fix the initial Cauchy hypersurface, and that the subsequent long term solution depends continuously on the initial data [17. Unfortunately, proving general global existence theorems is very difficult and currently not possible using standard mathematical techniques [12. The conformal method [17] is often used for investigating the Einstein field equations. In this case the free data are specified and the resulting constraints reduce to 4 elliptic equations. When the mean curvature is constant (see later), the constraint equations simplify even further to a linear system of 3 (elliptic) equations and a decoupled non-linear, scalar equation (the 
Lichnerowicz equation).

Due to the conformally invariant nature of the causal structure in a Lorentzian manifold, the compactified "regular conformal field equations" were constructed from the Einstein field equations, which constitute a first order symmetric hyperbolic system and consequently leads to well posed evolution equations and, in addition, global existence theorems for small data which follow from stability results for quasi-linear hyperbolic equations. In particular, Friedrich [18 proved a future global existence result for small initial data that is close to conventional data specified on a Minkowski hyperboloid. The conditions on generic initial data specified on an asymptotically flat Cauchy surface that subsequently leads to a Cauchy development with regular conformal completion is still not known. Friedrich has, however, pioneered an approach in which the conformal structure at spatial infinity is investigated $([19,20$, and new problems with regularity have been pointed out; (also see 21, 22 and references within).

Currently the only known global existence theorems for the full 4 dimensional (with no symmetries) field equations in GR are (i) the non-linear stability theorem of Minkowski space [23, (ii) the semi-global existence result for the initial value problem on a hyperboloid [18] and (iii) the semi-global existence result for spatially compact spacetimes with a hyperbolic Cauchy surface [24]. All of these results are for small data. It is known that vacuum (and electrovac) spacetimes containing a Cauchy horizon which is analytic and "ruled by closed null geodesics" admit a non-trivial Killing vector field [25]. There are many results possible when special spacetimes with symmetries are considered, and these results are briefly summarized below. Of course, a spacetime admitting a Killing vector fields is not generic, but such results may be interpreted in favour of a strong cosmic censorship theorem (see below).

Cases with symmetry: A global existence theorem is possible in special spacetimes with symmetry [12, 13]. A well known example is the global existence theorem for (smooth) spherically symmetric static solutions with perfect fluid or collisionless matter [12. The symmetry of a spacetime is characterized by the total number of Killing vector fields and their nature. Let us consider spacetimes with an $r$-dimensional Lie algebra of spacelike Killing vector fields. Then, for each $r$ (with $r \leq 3$ ), there are a number of results on global existence and various conjectures regarding cosmic censorship [13. In particular, when $r$ $=3$ (Bianchi models [26) and the special polarized Gowdy models with $r=2$ (see references below), the global behaviour of solutions of the Einstein field equations is reasonably well understood.

There are only partial global existence results in the general case of $r=2$ (with a local $U(1) \times U(1) G_{2}$ symmetry). In addition, there is no resolution of the cosmic censorship conjecture 13. The original global existence theorem for Gowdy spacetimes with $R \times T^{3}$ topology was presented in [27. Subsequently this theorem was generalized to spacetimes on $S^{3}$ and $S^{2} \times S^{1}$ [28 (a subclass of metrics that are not "generic" remain to be investigated). Theorems on vacuum Gowdy spacetimes with a global constant mean curvature foliation were 
first proven in 29]. Cosmic censorship has also been investigated utilizing the asymptotic behaviour of curvature invariants in the polarized Gowdy spacetimes [30] and more generality [31. Unfortunately, the horizon structure and extensions in polarized Gowdy spacetimes is extremely complex [32, 33. There are no large data global existence results nor theorems on cosmic censorship in the $r=$ 1 case $(U(1)$ symmetry) or $r=0$ (no symmetry) case. However, from numerical experimentation there are some conjectures on the generic behaviour in $U(1)$ spacetimes as well as small data semi-global existence results in the direction of expansion [34, 35].

Differentiability: Technical questions relating to differentiability are important for many of the mathematical problems described, and especially for wellposedness [12]. The smoothness of the Cauchy data for the initial value problem of a system of partial differential equations and that of the corresponding solutions are determined by the partial differential equations themselves. Indeed, there is a correspondence between the differentiability of the free data and the full data in the Einstein constraint equations.

But there is important motivation for assuming weaker differentiability conditions than the natural condition of $C^{\infty}$. For example, one reason is that the matter fields are not necessarily $C^{\infty}$ (so that results may not be applicable in physically important scenarios). Another reason is the potential for continuing local existence results of lower differentiability solutions to global existence results. In addition, there are also indications that generally Cauchy horizons may not be differentiable 36 .

Therefore, it is important to find a theoretical framework for investigating the constraint and evolution equations for spacetimes with milder differentiability (e.g., to prove results with lower differentiability; e.g., to consider metrics of regularity class $C^{1,1}$ [37] (where the first derivatives of the metric functions are locally Lipschitz continuous functions, which is perhaps a more appropriate class than the regularity class $C^{2}$ in many situations of physically interest). The initial data for the vacuum field equations can be relaxed further and assumed to belong to a local Sobolev space, especially in existence and uniqueness theorems. Indeed, in harmonic coordinates the vacuum field equations constitute a quasi-linear hyperbolic system of equations, and the usual theorems imply that the resulting Cauchy problem is well-posed in an appropriate Sobolev space [38] (see also [39, 40]).

Singularity theorems: The singularity theorems are an immense theoretical accomplishment within GR and, indeed, within mathematical physics in general [41]. Penrose's original singularity theorem [42] introduced the concepts of geodesic incompleteness (wherein geodesic curves exist that cannot be extended in a regular manner to the complete spacetime) to characterize singularities, Cauchy hypersurfaces and global hyperbolicity, and closed trapped surfaces [4]. These concepts have proven crucial to the development of mathematical GR. Subsequently, Hawking understood that closed trapped surfaces may occur in 
a Universe that was expanding to the past, which could then also lead to an initial cosmological singularity [44. This then led to the famous singularity Hawking and Penrose theorem [45]: if a convergence and a generic condition holds for causal vectors, and there are no closed time-like curves and there exists at least one of the following: a closed achronal imbedded hypersurface, a closed trapped surface, a point with re-converging light cone, then the spacetime has incomplete causal geodesics. From this theorem the discovery of the cosmic microwave background then implies that a singularity may of actually occurred in the past in our own Universe [46].

The singularity theorems of Penrose and Hawking show that, under reasonably general conditions, spacetime singularities may be inevitable [42, 45. However, these theorems do not say very much about the properties of generic singularities. There are also "general" spacetimes that do not have singularities [47; e.g., the analysis in the Penrose singularity theorem does not necessarily imply that a trapped surface will form during evolution. However, it was subsequently proven [48 that in vacuum spacetimes a trapped surface can dynamically form from regular initial data that does not have a trapped surface (this theorem was generalized in [49, 50]). A sequence of marginally outer trapped surfaces, in which the area approaches zero and hence forms an apparent horizon, were then constructed within a region up to the "center" of gravitational collapse in the full $4 \mathrm{D}$ vacuum GR field equations [51. The existence of marginally outer trapped surfaces are important for proving the positive mass theorem and the Penrose inequality [52] (see below).

There are many outstanding questions. These include obtaining more general singularity theorems with both weaker energy conditions and with weaker assumptions of differentiability, and investigating whether there is a relationship between geodesic incompleteness and the divergence of curvature invariants 47 . In addition, there are various related outstanding problems in mathematical cosmology. Generic spacelike singularities are often called cosmological singularities. But oscillatory singularities may also correspond to the spacelike part of generic black hole singularities [41, 47, since the mass inflationary instability at the inner horizon of a rotating black hole that is accreting may be followed by an oscillatory collapse to a spacelike singularity [53. There are also the questions of whether the singularities of GR can be resolved by quantum effects and whether singularity theorems are possible in higher dimensional spacetimes [1]. But perhaps the most pressing unresolved question within GR concerns the cosmic censorship hypothesis. 


\subsection{Cosmic censorship}

The theorems of Penrose and Hawking [42, 45] imply the existence of singularities. However, although the simple Schwarzschild spacetime does contain a singularity, the singularity is hidden inside a black hole event horizon and is thus not visible from the exterior region. Therefore, it is of interest to ask whether the gravitational collapse of physical matter leads to singularities like the Schwarzschild singularity in the sense that they are hidden inside an event horizon and they are not time-like (satisfying weak cosmic censorship and strong cosmic censorship, respectively [43]).

As a result, Penrose introduced [2] the cosmic censorship hypothesis. This asserts, roughly speaking, for the gravitational field equations of GR coupled to "physical" matter fields, that no "naked singularity" will develop from regular "general and realistic" initial Cauchy data. Essentially a naked singularity is a singularity from which light can escape to distant regions. Such a singularity poses severe problems, since the future is no longer predictable [46]. It is not reasonable to conjecture that such singularities can never occur, since there are examples of spacetimes that admit a naked singularity. However, such spacetimes are highly symmetric and it is not inconceivable that naked singularities formed under small (general, physically realistic) perturbations will be hidden by an event horizon. In fact, analysis implies that naked singularities only form in spacetimes with symmetry [29].

For example, naked singularities are known to exist in Taub-NUT spacetime 54, 33. In addition, so-called "shell crossing" will occur in a pressure-less fluid or "dust" spherically symmetric Lemaitre-Tolman-Bondi-de Sitter collapse 55, in which a central locally naked singularity will form from typical regular initial data and where various curvature scalars diverge. The most rigorous theorems are by Christodoulou [56, 57, who proved that naked singularities can develop from regular initial, asymptotically flat data for solutions of the globally inhomogeneous spherically symmetric Einstein field equations coupled to a massless scalar field. However, perhaps the work on critical behaviour by Choptuik [58] (see later) is more important.

Therefore, it is desirable to formulate cosmic censorship as a precise mathematical conjecture, and then seek a proof. The formulation of the conjectures by Christodoulou [59] are perhaps the most widely utilized, although the revised formulation of strong cosmic censorship given in the introduction of 48 corrects some flaws 60 in the original formulation given in [59]. Unfortunately, such theorems, which lie within the global theory of partial differential equations, are very difficult to prove [61. A simpler approach might be, since there are no time-like singularities in a globally hyperbolic spacetime, to attempt to to formulate (strong) cosmic censorship by asserting that spacetime must be globally hyperbolic under appropriate general conditions. Unfortunately, a maximal Cauchy development of initial data in a globally hyperbolic spacetime may not constitute the complete spacetime. An alternative approach is to seek a counterexample to any mathematical cosmic censorship conjecture.

There are two other particular issues. First, it is very difficult to mathe- 
matically define a naked singularity since the field equations are essentially hyperbolic and hence extending a solution to regions which are in causal contact with the singularity is problematic; consequently, we seek an alternative definition of a naked singularity that can be formulated mathematically. The second problem is genericity. It is not possible to prove a general theorem that naked singularities cannot exist since there are exceptional spacetimes which, for any reasonable definition, contain a singularity in which the maximal development is, in fact, extendible. Therefore a "genericity condition" is needed, otherwise such a statement of cosmic censorship would necessarily fail. However, we are ultimately interested in the physical process of gravitational collapse, and so we want to be careful not to formulate a conjecture that might admit an artificial counterexample. Consequently, we wish to formulate the cosmic censorship conjecture so as to avoid counterexamples are not physical, but at the same time not at the expense of making cosmic censorship impossible to disprove [12].

There are, in fact, both weak and strong cosmic censorship hypotheses, and these two hypotheses are only marginally related. The weak cosmic censorship hypothesis states that: For generic initial data to the evolution problem in GR, there cannot be naked singularities. It is of prime importance to prove the weak cosmic censorship conjecture. This is such an open problem that the correct formulation of the statement is not even known [59. This conjecture is discussed extensively in 62. The work of Christodoulou discussed above 63. essentially proves weak cosmic censorship for asymptotically flat, spherically symmetric Einstein-scalar field spacetimes. In addition, Christodoulou also presented examples of naked singularities that develop from initial Cauchy data [57]).

The strong cosmic censorship hypothesis states that: A generic solution to Einstein's field equations cannot be continued beyond the Cauchy horizon. For reviews of this version of the cosmic censorship conjecture see [64, 32. It would be informative to prove the two versions of cosmic censorship even for solutions of the vacuum field equations or, more generally, for classes of spacetimes with symmetry. In particular, it is of interest to prove that the maximal Cauchy development for generic vacuum data sets is equal to the maximal vacuum extension of a 3D compact manifold.

Alternatively, and as noted above, a different approach is to look for a counterexample to cosmic censorship. If a variety of classes of possible counterexamples can be shown to not to work, it might even be viewed as evidence for the likely veracity of the conjecture. Recently, a plausible vacuum counterexample to cosmic censorship with a negative cosmological constant has been proposed utilizing the superradiant instability of the Kerr-AdS black hole [65]. Another plausible 4D asymptotically anti-de Sitter counterexample modelling holographically an electrically charged localised defect within Einstein-Maxwell was presented in [66. In addition, this counterexample is generic [67, unlike the spherically symmetric collapse candidates which are finely tuned. 
The Penrose inequality The analysis used in the singularity theorems has been utlizied in several other problems in GR. For example, it has been applied in the positive mass theorem [68 and the study of rigidity in asymptotically flat spacetimes with non-negative scalar curvature. It was demonstrated by Penrose [3] that a spacetime obtained by evolving initial data will contain a naked singularity if a particular inequality is not satisfied. This inequality, called the Penrose inequality, relates the area of the so-called apparent horizon and the ADM mass within this marginally (outer) future-trapped surface. Consequently, initial data that does not satisfy this inequality could be used to construct a counterexample to the weak cosmic censorship conjecture. On the other hand, a "proof" of this inequality migth constitute evidence in favor of this form of cosmic censorship and might, in addition, lead to an analytical approach to attack the cosmic censorship conjecture 69, 13]). An important open problem is to prove the Penrose inequality or construct a generic counterexample.

Recently the Riemannian analogue of the Penrose inequality was proven 70. Unfortunately, there is no such proof in the Lorentzian case, even in simpler spacetimes with symmetry. For example, in spacetimes with spherical symmetry only a version of the conjecture using the energy rather than the ADM mass has been shown to be true. A number of proofs have been presented under a variety of different further assumptions such as, for example, the existence of constant mean curvature foliations [71) and global conditions on the spacetime 72. A formulation of the conjecture for certain null surfaces in spacetimes (the null Penrose conjecture) was studied in 73 .

In fact, the Penrose inequality is but one of a large number of mass inequalities for spacetimes 74]. E.g., a similar inequality exists utilizing the Penrose quasi-local mass 75 . In addition, it is of interest to generalize these mass inequalities to non-time-symmetric initial data. There are also stronger versions of the Penrose inequality that involve angular momentum and/or electric charge (or even a non-zero cosmological constant). All of these generalizations lead to additional open problems 41]. There are also additional refinements of some of the conjectures. For example, the Gibbons-Penrose inequality, gives a stronger lower bound if multiple black holes are present [76. There is also Thorne's hoop conjecture [77, which has been very useful 62. This inequality attempts to exploit the reasonable notion that the energy/matter content of a physical black hole, which is an extremely localized object, must be severely restricted in all spatial directions. A rigorous reformulation of the hoop conjecture was recently given [78. 
Critical phenomena: Choptuik [79] first studied the spherically symmetric gravitational collapse of a massless scalar field in GR numerically and found strong evidence for a universal, self-similar (critical) solution at the threshold of black hole formation. In particular, a one parameter family of solutions to the coupled Einstein-matter equations was considered. The continuous parameter $p$ was used to prescribe the initial data which, in turn, controls the strength of the matter which is initially undergoing gravitational collapse. For small values of $p$, where the strength of gravity is not appreciable, the spacetime remains regular everywhere (e.g., in the case of a massless scalar field the radiation will disperse to infinity). For large values of $p$, gravity is sufficiently strong that some of the matter becomes trapped within a singular black hole. For a special "critical" value of $p$, there exists a solution which is self similar and corresponds to the black hole formation threshold. Current studies suggest that almost every model containing black hole collapse will admit critical behaviour [58. It is an important mathematical problem within GR to better understand these critical solutions and to describe the corresponding critical behaviour in gravitational collapse in the general case in which there are no symmetries. Much work has now been done on critical phenomena in various models [58] including, for example, in Yang-Mills theory (see below). In [80] the authors claimed to have proven the existence of Choptuik's solution using computer assisted methods.

\subsection{The Einstein-Yang-Mills equations}

Yang-Mills (YM) fields over, for example, $R^{4}$ or $S^{4}$ are studied using techniques in partial differential equations. A number of important questions, including their mathematical stability and existence proofs, have not yet been resolved. A fundemental property of YM theory is its invariance under an infinite-dimensional group. The YM field equations depend on the "gauge" choice of a section of the Lie algebra valued bundle; in an appropriate gauge the YM equations become a quasi-linear elliptic system. SInce bundles, connections and curvature play a fundamental role in YM theory, it is also of interest to mathematicians 1. Numerical computations indicate that static, spherically symmetric Einstein-Yang-Mills (EYM) equations have non-singular, asymptotically flat solutions 81 .

In $4 \mathrm{D}$ there are smooth solutions of the YM equations globally for reasonably general initial conditions. Assuming sufficiently differentiable initial data, global existence was proven in Minkowski spacetime 82. Subsequently, local existence for data of finite energy was proven [83, and due to energy conservation this implies a global existence result. Global existence for $1+3$ dimensional, globally hyperbolic manifolds was proven [84, 31]). A global existence proof (to the future) for small initial data on a hyperboloid in Minkowski spacetime, which was subsequently generalized to Maxwell and YM matter, was presented in [85]. In addition, asymptotically flat, spherically symmetric "particle-like" solutions of the coupled EYM equations (with gauge group SU(2) and a regular interior) are known. However, their properties are not understood fully [86].

YM theory has also been investigated in higher dimensions $D$. For $D \geq 5$ 
solutions exist in which singularities develop in a finite time, although computations provide evidence this is stable in that the blow-up occurs for an open set of initial data. In addition, there are indications that this blow-up is separated from dispersion by a critical self-similar solution. However, there is no rigorous proof of blow-up, even in 5D. And in 6D, apparently different singularities (from those in 5D) can form [13.

Wave maps with values on spheres have also been studied, since they capture effects found in YM theory but in two less dimensions where theorems are easier to establish. In particular, a solution with the expected properties of the critical solution for wave maps in $4 \mathrm{D}$ in which a singularity forms was proven to exist 87 . (see below). A global existence theorem for the classical wave map equation is an important unresolved problem. There are small data global existence results for the non-linear ( $\sigma$-model, hyperbolic harmonic) wave map equation in space dimensions $n \geq 2$. Unfortunately, global existence results for large data are only known for solutions with symmetry. Even global existence for the wave map equation in $n=2$ has not been proven. However, utilizing energy estimates, global existence can be proven in the case $n=1$, [13, 88]. It is perhaps of interest to study the $\mathrm{U}(1)$ symmetric vacuum case in $4 \mathrm{D}$ in the presence of a spacelike Killing vector field which is hypersurface orthogonal. The Einstein field equations then simplify to 3D gravitational equations which are coupled to wave map matter, and so this case interpolates in difficulty between the $4 \mathrm{D}$ Einstein field equations and the Gowdy equations which are highly symmetric [89.

In particular, for YM theory in $n \geq 5$ and wave maps in $n \geq 3$ (from $D=$ $n+1$ dimensional Minkowski spacetime into the $n$-sphere) there are explicit selfsimilar solutions which are examples of finite-time singularity formation from regular initial data 90, (which are linearly stable; non-linear stability was proven later in [91]). The critical solutions sitting at the borderline between global regularity and blowup are self-similar and have exactly one unstable direction. Wave maps (from 3+1 Minkowski spacetime to the 3-sphere) are perhaps a particularly good toy model to study critical behaviour in gravitational collapse [58, although the critical solutions in this case are continuously (rather than discretely) self-similar. The dimensions $n=4$ for YM theory and $n=2$ for wave maps are critical and in these (and only these) dimensions there exist stationary solutions (the harmonic maps). The energies of these stationary solutions determine the thresholds for singularity formation (that is, solutions starting from initial data with lower energy are globally regular). It is also known that the process of singularity formation in these particular dimensions proceeds via shrinking of the stationary solution to zero (along the symmetry orbit generated by scaling). Note that in the case of wave maps the geometry of the target is important. For example, for negative curvature targets the wave map equation in $n=2$ has no stationary solution and consequently the evolution is globally regular. Later, new critical solutions were discovered in the EYM model (which evade Birkhoff's theorem and which already have rich dynamics even in the spherically symmetric case) 92 . 
Collisionless matter models: Alternative forms of matter such as, e.g., selfgravitating collisionless matter have also been considered [13, 12, 93. Global existence and uniqueness theorems for sufficiently smooth solutions of the classical Boltzmann (and Vlasov-Poisson) equations in Newtonian theory have been extended to GR. For example, the global existence of weak solutions and convergence of classical solutions with close to equilibrium initial data have been proven. And there are existence theorems for spacetimes with symmetry, such as static spherically symmetric, plane and hyperbolically symmetric spacetimes, and a subset of the general Gowdy spacetimes. Spacetimes undergoing spherically symmetric collapse have also been investigated. It is known that models with Collisionless matter can evolve globally without singularities. In fact, many models can be isotropic to the future. The general case has been investigated numerically [12, 13, but no analytical results are known. An open problem is to prove a global existence result for general (spatially inhomogeneous) collisionless matter models for small initial data and, in addition, to prove an existence and uniqueness theorem for spatially homogeneous Einstein-Vlasov models in the large initial data case. 


\subsection{Uniqueness and stability}

The proof of stability involves the mathematical study of the asymptotic behaviour of solutions of the highly non-linear Einstein system of partial differential equations, which is notoriously very hard. However, there are some special spacetimes of particular interest for which proofs exist. We note that de Sitter and anti-de-Sitter spacetimes will be discussed later.

Stability of Minkowski spacetime: Minkowski spacetime is known to be globally stable [94. The global existence (for small data) and the stability of Minkowski spacetime under the Einstein field equations was first investigated in [23, 94, where it was proven that if asymptotically flat initial data close to those of Minkowski spacetime are specified on a hyperplane for the vacuum equations, then the maximal Cauchy development of this initial data is geodesically complete. The asymptotic properties of such solutions have also been discussed. In particular, the possible behaviour in a suitable neighbourhood of infinity of asymptotically flat spacetimes, in which the initial matter distribution has compact support, has been investigated There have been a number of recent generalizations of these results [95]. The stability of Minkowski space for Einstein-Vlasov systems was studied in [96].

Uniqueness of black holes: If the final asymptotic state of any spacetime is conjectured to be either Minkowski spacetime or a black hole spacetime, then it is of interest to determine whether a given black hole is the unique stationary solution. The question of the uniqueness of non-vacuum steady state black hole spacetimes goes under the colloquial name of "no-hair" theorems. Neither of these problems have been completely resolved. However, the uniqueness of black hole spacetimes with additional symmetry, such as axial symmetry or rotational symmetry, has been established. The uniqueness of the GR 4D Schwarzschild and Kerr solutions was first was discussed in [46, and a theorem for Schwarzschild spacetime was established in [97, 98. The Kerr spacetime is the unique stationary regular vacuum solution (subject to some particular conditions) [99, and its uniqueness was proven in [100, 101]. The uniqueness of the non-vacuum rotating electrically charged Kerr-Newman black hole solution has not yet been proven in general [102, 103. In the case that a black hole spacetime is real analytic, then it is known to be unique. It is anticipated that this result is also true when regularity is relaxed to the assumption of infinite differentiability, but there are only some partial results known in this case. In particular, for a given stationary black hole spacetime there are no other approximate (under small perturbations) stationary solutions other than the given black hole solution itself. In addition, no other stationary exteriors are permitted when certain special structures on the event horizon are postulated. 


\subsection{Stability of Kerr spacetime}

If the Kerr-Newman black hole spacetimes do indeed represent the unique stationary states in GR, then a very important problem is to prove their stability (particularly under small perturbations). The stability of the Kerr metric was reviewed in [105] and discussed in [104. A particular goal is to demonstrate that perturbations of the Kerr solution (and, indeed, the Schwarzschild solution [106]) exponentially decay. Recently there has been substantial and exciting progress made in the linearised problem [107]. However, results for the full non-linear problem are still elusive.

A full, quantitative (i.e., which provides precise decay estimates) proof of the linear stability of Schwarzschild spacetime has recently been established by Dafermos, Holzegel and Rodnianksi in 107. In the passage from the linear to the non-linear stability of Schwarzschild new difficulties need to be overcome, some of which are similar to those encountered in the stability of Minkowski 94]. The non-linear stability of the Schwarzschild spacetime under the restricted class of non-trivial axially symmetric polarized perturbations was proven in [108. The new ideas introduced therein may be applicable in a more general setting and will hopefully be an important first step to proving the non-linear stability of Kerr to gravitational perturbations. Indeed, proving the non-linear stability of Kerr has become one of the primary areas of mathematical work in GR [109] (for further background see [110] and the important references to Dafermos and others cited within; perhaps the most concise and up to date survey can be found in the introduction of [11]).

Current observations are consistent with the predictions of GR, suggesting that the end point of mergers is a Kerr black hole. Indeed, all numerical computations provide support in favour of the non-linear stability of Kerr (and Kerr-Newman) black holes (at least for certain ranges for their angular momentum) [112. However, there is still no complete mathematical analysis of the stability of the generic Kerr black hole, nor a comprehensive understanding of its dynamics under arbitrary non-linear perturbations. A rigorous proof of the Friedman instability [113] (that for a real analytic, stationary and asymptotically flat spacetime with a non-empty ergoregion and no future event horizon, the energy of certain solutions diverge as time increases) was provided in [114] in the more general case of smooth but not necessarily globally real analytic spacetimes (only a unique local continuation condition was imposed, which always holds if the manifold is locally analytic but can also be inferred in the case when the manifold possesses an appropriate second Killing vector field). In contrast, Aretakis [115] has proven that axisymmetric extremal horizons (such as, for example, the extremal Kerr-Newman spacetimes) are unstable under linear scalar perturbations, unlike the subextremal case for which decay is known for all derivatives along the event horizon. 
The stability analysis has been extended to include a non-zero cosmological constant. Recently there have been results regarding non-linear perturbations in Kerr-de Sitter black hole spacetimes (at least in the slowly rotating case) [116]. However, an investigation in the case of a negative cosmological constant is much more difficult due to the potential superradiance instability of the KerrAdS black hole itself [117]). We shall discuss the stability of both the deSitter spacetime and the anti-de-Sitter spacetime below. An investigation of the stability of models which include matter is also important, particularly in the cosmological context (see later). Unfortunately, generalizations to even include a simple inhomogeneous perfect fluid are very difficult mathematically due to the possible formation of shocks and (in the case of dust) shell-crossings. It would first be necessary to further develop appropriate criteria to detect, using classical hydrodynamics, the possible development of shocks. 
The Final State Conjecture: The final state conjecture, originally discussed by Penrose in [1, is the statement that generic asymptotically flat and vacuum initial data (which can include black holes, and hence technically also matter if it's confined to the interior of the black holes, but no naked singularities) evolve to a solution which either disperses (in which case there are no black holes) or else eventually asymptotes (in the exterior) to finitely many Kerr solutions (a collection of unbound black holes) moving away from each other. That is, for small perturbations of Kerr, not only is null infinity still complete, but in the exterior region the Kerr metric is asymptotically stable (up to a redefinition of the parameters). The final state conjecture includes the statements of the non-linear stability of the (exterior of) Kerr spacetime [118] and weak cosmic censorship as special cases. It is of importance to prove this conjecture, but it is unlikely that this will be done in the near future [60.

If the metric is real analytic, as in the original formulation of the no-hair theorem, then the assumption of closeness to Kerr in the stationary uniqueness theorem is no longer necessary [46. However, this assumption of analyticity is not well motivated, since stationarity does not guarantee that the Killing vector field is timelike everywhere in the exterior (e.g., in the Kerr geometry itself, in the so-called ergoregion outside the black and white holes the Killing vector field becomes spacelike), and analyticity only follows from the ellipticity of the reduced equations in this case. If the analyticity assumption were weakened to smoothness only, then the consequent uniqueness property would support the more ambitious Penrose final state conjecture for vacuum spacetimes arising from generic asymptotically flat Cauchy data (not necessarily initially close to Kerr), whereby the Kerr exterior would not only be a stable endstate, but it would also be the general endstate for all non-dispersing vacuum solutions.

The final state conjecture includes not only the non-linear stability of Kerr but also the evolution of (generic) arbitrarily large amplitude gravitational waves. Consequently, by seeking quasi-normal modes in black hole mergers (i.e., with no hair) in gravitational wave observations the dynamics of GR and hence the final state conjecture is investigated. Therefore, this very important open question in mathematical GR is directly related to gravitational wave observations. Some evidence in favor of the final state conjecture has been provided by numerical simulations [119]. 


\subsection{Other problems}

Newtonian limit: A precise mathematical statement that within GR the limit of a divergent speed of light leads to Newton's theory of gravity is problematic. A formulation of a suitable definition of the Newtonianian limit of GR with physically reasonable properties was first presented by Ehlers [120]. Unfortunately, it is not clear that there are general families of solutions of the field equations within GR which have a Newtonian limit with the definition adopted. In addition, a mathematical justification of the "post-Newtonian" approximation relies on the existence of suitably differentiable families of such solutions. In particular, Bartnik's problem RB21 [4] seeks a proof that solutions of the Einstein field equations reduce to solutions of Newton's theory in a rigorously defined limit . And problem RB20 4] addresses the question of the range of validity of both the post-Newtonian and the post-Minkowskian asymptotic expansions. There has been much work done on finding solutions in which the metric satisfies the Einstein field equations approximately, consisting of both numerical computations and analytical techniques such as asymptotic expansions, linearisation techniques and matching analyses.

Geodesic hypothesis: An important postulate of GR is that the intrinsic geometrically defined spacetime geodesics are the trajectories along which point particles with negligible mass move. It remains to rigorously prove that such test particles move on geodesics in spacetime. This problem (RB23) was considered by Einstein himself and, despite extensive investigations using asymptotic expansions [121], has not yet been fully resolved. An essential obstacle is a rigorous definition of the "negligible mass limit". In addition, within GR a physical object itself causes a gravitational backreaction in the spacetime due its own motion.

Constant mean curvature: There are a number of problems in mathematical GR, involving both the initial data constraints and the evolution equations, in which the results obtained rely upon the underlying spacetime admitting a constant mean curvature (CMC) Cauchy surface (or slice). However, not all spacetimes have CMC slices [122] (although the known examples are very special with a high level of symmetry), and this has important ramifications. Consequently, perhaps the most serious question concerning CMC slices is their generality. If spacetimes generally admit CMC slices then they can be used for proving generic properties, and the special examples where they do not exist can be neglected. In [123, the known results were reviewed and a suite of conjectures concerning the existence of CMC slices and their generality were made. 
Scalar curvature invariants: It is known that a 4D (and higher dimensional [125]) Lorentzian spacetime can be completely characterized by its scalar curvature invariants (built polynomially from the Riemann tensor and its covariant derivatives), unless it belongs to the special class of "degenerate Kundt" spacetimes [124. The special class of Kundt spacetimes are those that admit a geodesic, expansion-free, shear-free, twist-free null vector. Therefore, in general, a Lorentzian spacetime is completely locally characterized by its scalar polynomial curvature invariants. A Riemannian manifold is always characterized by such scalar invariants.

The "inverse" problem of determining when a spacetime manifold can be explicitly constructed from its scalar curvature invariants is of interest. It is also important to find the minimal set of such invariants. In particular, to determine the minimal set of scalar curvature invariants needed for the explicit characterization of a non-degenerate 4D Kundt spacetime. We note that the Petrov type of the Weyl tensor in $4 \mathrm{D}$ is partially characterized by its scalar polynomial curvature invariants [126].

Horizon evolution: There has been a lot of work done in trying to formulate an appropriate definition of the "boundary of a black hole". If the evolutions along the two future null vector field directions normal to a closed oriented spacelike 2-surface (isomorphic to $S^{2}$ ) in a spacetime are both area-non-increasing to the future (or one of the null mean curvatures is zero), then that 2-surface is said to be future trapped (or, respectively, an "apparent horizon"). It is a particularly important open problem to formulate an appropriate definition (and hence a local characterization) of a dynamical horizon for an evolving black hole. A lot of the analysis of the evolution of apparent horizons is in the linear regime. This implies that to first order the horizons do not move. The non-linear evolution of apparent horizons is still not understood. Recently scalar curvature invariants have been utilized to identify and locate ("geometric") horizons [127.

Gravitational wave memory: The study of asymptotia, including that in non-asymptotically flat spacetimes (such as de Sitter spacetime) is also of importance. In particular, such issues arise in the problem of gravitational wave memory. GR describes gravitational radiation from astrophysical phenomena [128. Gravitational wave memory is a permanent displacement of a gravitational wave detector after the wave has passed, and has been extended to the full non-linear theory of GR by Christodoulou [129]. It is known that the memory found in an (ordinary) linearized situation (due to fields that do not reach null infinity) and (null memory) in the non-linear theory (due to fields that do reach null infinity) are two different effects. The ordinary memory is very small, whereas the null memory is sufficiently large to be detected by Advanced LIGO and LISA as a permanent displacement of test masses, and also in radio telescopes like NANOGrav as a change in the pulse frequency of pulsars. It is also known that electromagnetic fields and neutrino radiation enlarge the memory effect [128. 
Much of the work treats gravitational memory in an asymptotically flat spacetime. However, we appear to live in an expanding Universe, not an asymptotically flat spacetime. Moreover, the sources of gravitational waves are rare and those that have been detected to date have been at distances at which the expansion of the Universe cannot be neglected. As the detectors become even more sensitive, detections due to sources at even greater distances might be anticipated in which the expansion of the Universe will be even more important. A proper treatment of memory in an expanding Universe is thus crucial. Gravitational wave memory in the case where the sources and detector are in an expanding $\Lambda \mathrm{CDM}$ cosmology was examined in [130. The Universe is highly inhomogeneous. However, for simplicity, a de Sitter cosmological spacetime was assumed and the gravitatational radiation was treated in the short wavelength approximation. Results very similar to those of gravitational wave memory in an asymptotically flat spacetime were found, except that the total magnitude of the memory effect is amplified by a redshift-dependent factor and can be affected by gravitatational lensing.

Alternative theories of gravity: Many of the problems discussed here can be rephrased in the context of alternative (to GR) gravity theories. Such problems are outside the scope of the present article, but would include several questions within cosmology (see later and [131]). For example, it might be of interest to study the stability of the isotropic Friedmann model 132 on approach to an initial cosmological singularity in alternative theories of gravity. In particular, this question has been investigated in gravity theories with higher-order curvature terms in the Einstein-Hilbert action [133]. 


\section{Broadening the scope}

\subsection{Quantum gravity}

The attempt to formulate a fundamental theory which unites the standard model of particle physics, which includes the three forces of electromagnetism and the strong and weak nuclear forces, together with gravity (i.e., GR) into a single, fully consistent, unified theory of quantum gravity (QG), is a primary goal of theoretical physics.

GR is modified by QG effects, and new gravitational physics are possible. However, it seems that such modifications do not dramatically influence the macroscopic behaviour of stellar systems and black holes over astrophysical timescales 135. Perhaps the most spectacular consequence of the unification of GR and quantum mechanics is that a black hole evaporates due to the emission of Hawking radiation [134, but even here the classical properties of the black hole are not affected significantly. However, attempts to unify quantum phenomena and GR, such as string theory or loop QG, have motivated a number of new theoretical problems.

AdS/CFT correspondence: The unique maximally symmetric Lorentzian spacetime with constant negative scalar curvature in any dimension is Anti-de Sitter (AdS) spacetime. Such spacetimes are important in theories of QG formulated in terms of string theory or modern extensions such as M-theory. In fact, AdS spacetimes currently are of primary importance in theoretical physics due to the conjectured Maldacena AdS/CFT correspondence. This is a hypothetical fundamental equivalence between string theory on an asymptotically AdS spacetime and a conformal field theory (CFT) on its boundary [136, 137. CFT are conformally invariant quantum field theories describing elementary particles, and include theories of Yang-Mills type.

Gauge/gravity duality postulates that a quantum mechanical force such as electromagnetism (or forces arising from the weak or strong interactions) can be described by a string theory in an AdS spacetime with an additional dimension. Since AdS/CFT correspondence implies a non-perturbative formulation of string theory (with particular boundary conditions), it could potentially lead to significant advances in our comprehension of string theory and QG. And, of course, the utility of this gauge/gravity duality is that strongly coupled CFT can consequently be investigated by considering the corresponding weakly interacting theory of gravity, which may be more tractable mathematically. A number of problems in nuclear and condensed matter physics (including the modeling of heavy ion non-equilibrium collisions) have been investigated by translating those problems into mathematical problems in string theory. Namely, a AdS/CFT dictionary is utilized to translate a strongly coupled CFT to its string dual. The corresponding classical AdS gravitational theory is then investigated, and potentially useful results in the CFT are then deduced. The down side of this holographic approach is that the gravitational theory in the non-stationary sector is not always understood. 


\subsection{Instability of Anti-de Sitter spacetime}

The AdS/CFT correspondence provides strong motivation for studying asymptotically AdS spacetimes. Of course, the dynamics of AdS spacetimes within classical GR is itself of interest. Minkowski and de-Sitter spacetimes [138. were proven to be non-linearly stable many years ago 94,139 . However, this is not the case for AdS spacetime and, in fact, it has very recently been postulated that the AdS spacetime is not stable to (arbitrarily) small perturbations [140], By instability we mean that for large classes of initial data any perturbation of AdS spacetime, no matter how small, leads to black hole formation.

Prior to the work of [141, there was no real conjecture of instability of AdS (however, see [142]). In particular, the note 140 simply observes a physically obvious fact (proved in 143) that a perturbed AdS spacetime cannot settle down to a globally regular stationary asymptotically AdS spacetime because the only such spacetime is AdS itself (and the energy is conserved for reflecting boundary conditions). However, this is no argument for instability (and even less for black-hole formation) because the evolution can be a globallyregular-in-time quasi-periodic perturbation of AdS [143]. Perhaps the most important subsequent result was the perturbative construction of time-periodic asymptotically AdS solutions (with negative cosmological constant in an $n+1$ dimensional spherically symmetric spacetime) and numerical evidence of their instability [144].

The study of the global non-linear stability of AdS was given great impetus by the recent work of Bizon and Rostworowski 67 in an analysis of a spherically symmetric massless scalar field within GR. It was suggested by numerics that AdS is non-linearly unstable to a weakly turbulent mechanism. Arbitrarily small black holes can form. The mass of such black holes seems to be determined by the initial data. And although this non-linear instability appears to be present for a variety of typical perturbations, there are many perturbations for which such an instability is not generated [145. This consequently appears to suggest the existence of islands of stability [146. Indeed, understanding which configurations lead to instability and those that do not is a crucial question, and many analytical and numerical tools have been deployed to disentangle stable from unstable initial data in the search for islands of stability [147. 
In particular, secular growth and non-linearities were shown to occur that can then lead to resonances and weak turbulent behaviour 67. Heuristically, this turbulence is believed to be triggered by the generation of secular terms by resonant four-wave interactions which drive the instability. Perturbations to third order in the amplitude of the linear seed were used in the analysis. Modifications of standard perturbation theory, such as the resonant approximation [141, can then be used to represent the dynamics for particular time scales. However, these approximations depend on spherical symmetry. It is not currently known if any GR solution with a fully resonant spectrum must lead to a non-linear instability; however, it appears that such a condition is necessary for the existence of such a weakly turbulent instability. The role of the fully resonant spectrum remains unclear 145; but some results do not rely on any resonances 148. It is of interest to determine if the non-dispersive character of the linearized spectrum is necessary for the turbulent instability. It is also of interest to investigate the generality of such an instability.

It is of great interest to investigate this further without the assumption of spherical symmetry. A number of different seeds have been used in calculations to third order in perturbation theory [145, 146], and it appears that the gravitational possibilities are richer than in the spherically symmetry case studied previously [67. It is, of course, essential to investigate the non-linear stability of AdS without any assumptions on symmetry. However, despite some recent research [147, such an investigation is currently not possible, neither from an analytical nor even a numerical standpoint. It is therefore of primary importance to determine the possible end point of instability for non-spherically symmetric perturbations for AdS spacetimes in any dimension [140. That is, to determine if the conjectured non-linear AdS instability has different properties in more general spacetimes than those in spherically symmetric scalar field collapse. And, in addition, to determine whether this conjectured non-linear instability leads to a weakly turbulent mechanism that subsequently develops a "cascade" towards higher frequencies and the formation of black holes. This is discussed in more detail in [149] (also see the review [147]). We remark that non-linear instability was recently proven for the Einstein-massless Vlasov system in the case of spherical symmetry [148]. 


\subsection{Higher dimensions:}

Additional spatial dimensions (to the usual three of normal space) are utilized in string theory [150. Higher dimensional spacetimes are also considered in a number of scenarios within cosmology. If, indeed, higher spatial dimensions exist, then fundamental questions on the character of the internal space of the Universe arise.

The investigation of black holes in higher dimensional GR is particularly important. Classically gravity, and especially black holes, has a much richer structure in higher dimensions than in standard 4D. In particular, it is known that the stationary Kerr black hole is unique in 4D GR. However, there are are a variety of asymptotically flat, exact vacuum black hole solutions in higher dimensions 151. An important open question is to determine the uniqueness and stability of black holes in higher dimensions. This leads to a number of interesting new problems, including the reformulation of the hypotheses of analyticity, non-degeneracy and connectivity in the study of uniqueness of black-holes in higher dimensions. It is also of interest to classify all vacuum "near-horizon" geometries with compact cross-sections.

Differential geometry has recently been further developed in higher dimensions [124, 152. In particular, despite the fact that originally the singularity theorems were investigated in $4 \mathrm{D}$, there exist results for co-dimension two closed trapped surfaces in any arbitrary dimension. In fact, the definition of being trapped can be extended to submanifolds of any co-dimension (although a suitable condition on the curvature must be postulated in order to guarantee that focal points to the submanifold exist). In particular, co-dimension three closed trapped submanifolds in higher dimensions were studied in [153. And recently, a positive mass theorem has been presented in arbitary dimensions [154].

It is also of interest to investigate the question of stability in higher dimensions. However, this is a much more complicated question since while the radiative decay of solutions is greater in higher dimensions, which would enhance stability, there is also a larger number of degrees of freedom, which, in general, would increase the potential for an instability. We note that numerical evidence does suggest that a number of higher dimensional black holes are unstable 151.

Cosmic censorship in higher dimensions is a very difficult problem and, indeed, is not even a well posed problem. In fact, there is a lot of numerical evidence that higher dimensional black holes can be unstable under gravitational perturbations, so that cosmic censorship consequently fails [155]. This was originally demonstrated for black strings and p-branes 156] (in 4D such an instability is not possible). There is numerical evidence [155] that black strings are unstable and "pinch off" in a finite time, thereby resulting in a singularity that is naked. This constitutes a violation of weak cosmic censorship because the initial data is not finely tuned. However, these spacetimes have compact additional spatial dimensions. The black rings of [157] also suffer from this and various other types of instabilities [158]. 
Rapidly spinning black holes deform significantly and are not, in fact, dissimilar to black branes. Myers-Perry (MP) black holes [159] (which are higherdimensional analogues of Kerr black holes) with large angular momenta have been shown to exhibit an "ultraspinning" instability when the spacetime dimension exceeds six [160]. In particular, the final point of the instability of asymptotically flat axisymmetric 6D MP black holes was investigated and the ultraspinning instability was shown to produce a "sequence of concentric rings which are connected by segments of black membrane on the rotation plane" [161. In the non-linear region these concentric rings become ever more thin, and in a finite time a naked singularity forms resulting in a violation of weak cosmic censorship.

There is also interest in theoretical results such as, for example, the dynamical stability of higher dimensional cosmological models. Spatially homogeneous cosmologies in higher dimensions and, in particular, extensions of the BKL analysis have been investigated [162. 


\subsection{Cosmology:}

Cosmology is concerned with the large scale behaviour of the Universe within GR (or, in some cases of interest, a particular alternative theory of gravity). There are a number of problems in mathematical or theoretical cosmology of current importance.

Cosmological inflation is generally accepted to resolve the horizon problem, that the Universe seems to be more uniform than expected on large scales. However, it should be noted that alternative explanations for this might be possible. The are many theoretical problems with inflation, which include the precise nature of the hypothetical inflaton field and the details of cosmic inflation. In particular, does the amplification of quantum-mechanical fluctuations lead to self-sustaining inflation and is it consequently still occurring in various places in the Universe. In addition, it is anticipated that initial conditions in both the inflaton field and the geometry contain inhomogeneities. But inflation does not necessarily occur for random initial conditions. For example, it has been demonstrated that small field inflation is sensitive to inhomogeneities [163. However, large field inflation appears to be more robust to inhomogeneous and anisotropic initial conditions, particularly in the simple case that initially any large energy gradients in the inflaton field are confined to the inflationary portion of its potential. An open problem is to determine the generality of inflation for generic initial data.

Additional questions include, for example, the beginning and late time behaviour of the Universe, and especially whether the Universe is evolving towards a final singularity. Using a modified theory of gravity (i.e., an alternative to GR) or invoking non-standard material fields, it has been shown that the final or initial singularity can be replaced classically by a bounce to another separate expanding region [164, 165. Therefore, it can be asked whether spacetime evolves towards a "big" bounce and, indeed, whether it is part of an infinite cyclic Universe.

Cosmology is concerned with the large scale dynamical behaviour of the Universe in which small-scale structures (e.g., those of galaxies) are negligible. Therefore, it is usually assumed that the "Cosmological Principle" is satisfied. This principle asserts that on large scales the Universe can be accurately modeled by an isotropic and spatially homogeneous solution to the Einstein field equations. Namely, this states that a preferential cosmological time can be chosen so that at all times, and on large scales, space appears the same in all directions (isotropy) and at all places (spatial homogeneity). The Universe is obviously not spatially homogeneous on the scales of galaxies or clusters of galaxies. Consequently it would be desirable if the cosmological principle (that is, late time spatial homogenization and isotropization) could be deduced from the field equations of GR under suitable physical conditions and for appropriate initial data, rather than postulated a priori. This is perhaps addressed within inflation, at least to some extent. 
The cosmological constant problem and dark energy: The most important problems in physical cosmology concern dark matter and dark energy [166. These forms of matter and energy cannot be directly observed and can only be identified by their gravitational effects. The existence of dark matter in the Universe is deduced from observed galaxy rotation curves, nucleosynthesis analyses and structure formation computations [167. The form of the missing dark matter is not yet known. In fact, it is not even known if it is a particle or whether the dark matter phenomena can be described by a generalization of GR. It is, however, believed that this problem will be resolved by standard physics. The dark energy problem, on the other hand, is regarded as a primary impediment to further understanding within theoretical physics [168, [169].

The cosmological constant problem has been discussed in detail by Weinberg 170. Conventional quantum field theory (QFT) leads to an exceedingly large vacuum energy density. However, the GR equivalence principle asserts that all forms of energy density have the same gravitational effect; hence it is assumed that the vacuum energy gravitates in exactly the same way as a cosmological constant, and consequently has a huge effect on spacetime curvature. But the observed effective cosmological constant is exceedingly small compared with the predicted value from QFT, so that a putative "bare" cosmological constant would be necessary to cancel out the enormous $10^{120}$ contribution from the vacuum. This is an unbelievably difficult fine-tuning problem. And it gets worse still when higher loop corrections are taken into consideration [171. Weinberg and other physicists have advocated that, out of all of the possible suggestions to resolve this problem, perhaps the most reasonable is the controversial argument of an anthropic bound 172 .

Moreover, for the last few billion years the expansion of the Universe has been increasing [173. The reason for this acceleration is commonly referred to as dark energy within the standard cosmological model, which has similar dynamical properties to a relatively small cosmological constant. The so-called coincidence problem of explaining why the cosmological constant has such a tiny observed value of the order of magnitude of the current matter density in the Universe must also be addressed. It has been conjectured that dark energy is not a simple cosmological constant but is, rather, a dynamical field due to, for example, quintessence or phantom energy. Some researchers have also put forward modifications to gravity on cosmological scales to explain the dynamical gravitational effects attributed to dark energy. Another possible explanation proposed for the observed acceleration of the Universe is backreaction from inhomogeneities, that occur when averaging Einstein's field equations on large scales.

\subsection{Averaging:}

The (effective) dynamical gravitational equations on cosmological scales are presumably derived from an averaging or coarse graining of the Einstein field equations. Averaging in cosmology is a problem of considerable interest, and its resolution is necessary for the correct interpretation of cosmological obser- 
vations. Although the so-called standard $\Lambda \mathrm{CDM}$ cosmological model, which is a spatially homogeneous and isotropic Friedmann-Lemaitre-Robertson-Walker (FLRW) spacetime with a cosmological constant $\Lambda$, makes predictions in exceptional agreement with current cosmological observations (except for a relatively small number of possible tensions [174]), it does require a dark energy that dominates the dynamics of the present Universe which, as noted above, has never been detected directly. Moreover, the current Universe is neither isotropic nor spatially homogeneous locally. Indeed, observations indicate that the current structure of the Universe is very complicated. Groups and clusters of galaxies of varying size form the largest gravitationally bound structures. These clusters themselves then "form knots, filaments and sheets that thread and surround very underdense voids". An enormous "cosmic web" is consequently created [175. An important open question in cosmology is whether averaging of inhomogeneities can lead to significant backreaction effects on very large scales.

A crucial first step is, of course, to formulate a rigorous mathematical definition of averaging in GR. Any such spacetime volume averaging procedure must necessarily be both well-posed and covariant, and this introduces some fundamental new issues within differential geometry. The mathematical problem of formally averaging tensors and other geometric objects on a differential manifold has recently received renewed attention [176, 177. The field equations obtained by averaging (or, more precisely, coarse graining) do not necessarily even have the same mathematical structure as the underlying Einstein field equations. For example, in the macroscopic gravity approach to spacetime averaging [177, which utilizes the Lie-dragging of averaging regions and is both fully covariant and gauge invariant, the structure of the averaged spacetime is not necessarily even Riemannian. However, the macroscopic gravity approach does lead, in general 176, to well-defined local averaged geometrical objects on an arbitrary Riemannian manifold. In particular, the averaged structure equations of the underlying geometry yields an appropriate set of structure equations for the averaged (macroscopic) geometry. In addition, macroscopic gravity gives the form for the additional terms (the so-called correlation functions) arising from the averaging of the non-linear field equations that necessarily occur in the macroscopic field equations [177]. 


\subsection{Stability of de Sitter spacetime}

Rigorous mathematical results are possible in the study of the stability of cosmological solutions. This involves an investigation of the behaviour of a complex set of partial differential equations at late times about a particular cosmological solution (and there are a number of exact solutions that are of special cosmological interest [24, 178]). First, we recall that if the cosmological constant is zero and assuming that the matter fields satisfy the standard energy conditions, then Bianchi type IX spacetimes recollapse and are consequently not expanding to the future for all times. Formally this result is encapsulated within the closed universe recollapse conjecture [179], which was proven in [180. However, in the case of a non-zero positive cosmological constant, Bianchi type IX spacetimes do not necessarily recollapse to the future. In particular, the question of the stability of de Sitter spacetime is of primary importance.

A stability theorem for de Sitter spacetime that utilized regular conformal field equations was proven in [181. Indeed, Friedrich proved that all (vacuum) initial data on a regular Cauchy hypersurface sufficiently close (in an appropriate Sobolev topology) to the initial data corresponding to a de Sitter spacetime with a positive cosmological constant have maximal geodesically complete Cauchy developments. In addition, the analysis by Friedrich also presented details of the resulting asymptotic behaviour of the solutions. Consequently, de Sitter spacetime acts as an attractor for expanding cosmological models with a positive cosmological constant, and the proof may be viewed as justification of the "cosmic no hair" conjecture (at least in the vacuum case; see also 22] and references within).

A cosmic no hair theorem for spatially homogeneous cosmological models was proven by Wald [182]. This general theorem, that shows that an expanding anisotropic cosmology which does not subsequently recollapse asymptotes to an isotropic de Sitter spacetime to the future, does not depend on the particular nature of the matter fields present other than that they satisfy the strong and dominant energy conditions. It can also be demonstrated that initially expanding solutions for initial data induced by the Einstein field equations with a positive cosmological constant and coupled to various reasonable matter models exist globally in time [183. In particular, global results are possible for inflationary scalar field cosmological models with an exponential self potential in which the rate of expansion increases polynomially with cosmological time [184, 185. We note that inflationary behaviour also occurs for scalar field models with a power law self potential, but this arises at intermediate times and not to the asymptotic future. Local dynamics are mainly studied numerically, and it is difficult to establish rigorous local results. It is an important open problem to prove a cosmic no-hair theorem in generic spatially inhomogeneous spacetimes. Some partial results are known for spatially inhomogeneous models with a positive cosmological constant [186] and, to a lesser extent, for scalar field cosmologies with an exponential self potential [184]. 


\subsection{Cosmological singularities}

Although standard theorems inform us that singularities generically occur within GR, they give very few details about the character of these singularities [4]. Belinskii, Khalatnikov and Lifshitz (BKL) [187] have postulated that the approach to a spacelike singularity to the past is "vacuum dominated, local, and oscillatory" (or "mixmaster") for general inhomogeneous GR cosmological models. In particular, BKL conjectured that due to the non-linearity of the Einstein equations, if the matter fields do not include a massless scalar field or its dynamical equivalent then in a neighbourhood of the initial cosmological singularity all of the matter fields can be neglected relative to the dynamical anisotropy in the field equations. The assumptions and associated dynamics of BKL have been demonstrated to be compatible with the field equations of GR. However, that does not necessarily imply that the dynamics are generally valid in situations of physical interest. Numerical simulations do support the validity of the BKL conjecture, at least for particular classes of spacetimes [188, 189].

There have been a number of different approaches to study the general structure of cosmological singularities. These include the more heuristic BKL metric and Hamiltonian approaches and the mathematically more rigorous dynamical systems approach [190]. In the dynamical systems approach the Einstein field equations (not necessarily with any symmetries) are reformulated as a scale invariant asymptotically regularized first order system of autonomous ordinary (or partial) differential equations valid in the approach towards a generic spacelike cosmological singularity 191. In more detail, in this reformulation the Einstein field equations split into a decoupled equation for a conformal factor and a coupled system of equations for the associated dimensionless conformal metric, where the conformal factor is related to the expansion of the normal congruence to an assumed foliation of spacelike surfaces in a sufficiently small neighborhood of a generic "simultaneous" spacelike singularity [192, 191]. This approach has facilitated a detailed description of the general attractor and led to precisely formulated mathematical conjectures regarding the general asymptotic dynamical behaviour towards an initial singularity. The reformulation also provides a suitable foundation for the numerical investigation of generic cosmological singularities [13].

Only a few rigorous mathematical results had been proven until quite recently. Following earlier analysis [193, Ringstrom presented important results on the asymptotic dynamics of spatially homogeneous cosmological models within GR [194. Indeed, Ringstrom proved theorems concerning the oscillatory behaviour of generic initial spacelike cosmological singularities for models of Bianchi types VIII and IX. In particular, Ringstrom demonstrated that the past attractor in a Bianchi type IX spacetime is contained within a union of vacuum subsets of Bianchi type I and Bianchi type II. Unfortunately, the attractor is not fully specified by this theorem [192. In addition, the results do not completely determine the relevance of the Kasner map for the dynamics asymptotic to the initial singularity in the Bianchi type VIII and IX models [195. 
The emphasis to date has been on proving mathematical theorems for spatially homogeneous cosmological models. The dynamics of Bianchi type IX models are still not fully understood. In [192] a summary of the asymptotic dynamical behaviour of Bianchi type IX models that has actually been proven was presented (also see [195]). For example, arguments concerning the possible chaotic behaviour at a generic spacelike singularity rely on the fact that the Kasner map actually describes the asymptotic dynamics of the Einstein field equations. However, although it is plausible that the Kasner map, which is indeed associated with chaos, plays such a role, this has not yet been proven 192. There remain a number of outstanding questions, which include rigorously establishing the role of Bianchi type IX models in generic cosmological singularities 196, 191, 192. In particular, an important open problem is to prove that the past attractor of the Bianchi type IX dynamical system coincides exactly with (rather than being a subset of) the Mixmaster attractor [192, 194]. Based upon a numerical and qualitative analysis [198, the exceptional Bianchi type $\mathrm{VI}_{-\frac{1}{9}}$ class B model (which is of the same generality as the most general Bianchi type VIII and Bianchi type IX class A models) also has an oscillatory initial singularity.

In cosmological models with matter, the BKL oscillatory dynamics have primarily been investigated in perfect fluid models with a simple linear equation of state relating the fluid pressure and density. However, some other matter fields can have an important dynamical effect in a neighbourhood of the initial singularity. A (massless) scalar field (or, equivalently, a stiff perfect fluid) will lead to the complex oscillatory dynamical behaviour being replaced by simple monotonic dynamical behaviour on approach to the spacelike singularity [197. On the other hand, an electromagnetic field can produce oscillations which are not present in vacuum or perfect fluid models of the same type (e.g., models of Bianchi types $\mathrm{I}$ and $\mathrm{VI}_{0}$ ) [199. And oscillatory behaviour can also occur in all tilting perfect fluid spatially homogeneous cosmologies [200, 201].

It is very important to investigate generic initial spacelike singularities in spatially inhomogeneous cosmological models and, in particular, to prove the BKL locality conjecture. Using Hubble (or expansion)-normalized variables, both qualitative and numerical evidence provides support for BKL oscillatory dynamics, at least for an open set of time lines [202]. More generally, a physical argument to justify asymptotic locality is that extremely strong gravitational effects will lead to particle horizons shrinking to vanishing size as the initial singularity is approached along an individual time line, so that communication between different time lines may not be possible ("asymptotic silence") in the asymptotic limit. In order to gain additional insight into general cosmological singularities in spatially inhomogeneous spacetimes, $G_{2}$-models with two commuting spacelike Killing vector fields have been studied. Oscillatory BKL dynamics has been discussed in general vacuum, spatially compact $U(1) \times U(1)$ spacetimes with zero twist and in generic polarized $U(1)$ spacetimes [13, and in twisting $U(1) \times U(1)$ vacuum Gowdy models on $T^{3}$, and on $S^{2} \times S^{1}, S^{3}$ and lens spaces [203]. The attempt to describe the dynamics in the asymptotic approach 
towards a generic cosmological singularity in terms of an attractor has led to a number of precise mathematical conjectures being formulated [196, 191]; indeed, the existence of a possible finite dimensional attractor in the infinite dimensional state space has been suggested 204.

A Hamiltonian approach (related to the BKL approach) has been utilized to heuristically investigate the dynamical behaviour of the Einstein-dilaton-pform system in the asymptotic approach to a spacelike singularity [205]. The limiting dynamics of the system was described in terms of a "billiard" motion in a subset of hyperbolic space bounded by "walls" (this dynamical behaviour was dubbed "cosmological billiards"). A remarkable mathematical connection between the resulting asymptotic dynamics and Kac-Moody algebras was found 205. The relationship between this approach to studying spatially inhomogeneous cosmologies and the more rigorous dynamical systems approach was presented in [196. The fermionic sector (including classical gravitinos) of supergravity theories was also recently studied [206]. A quantum extension of fermionic cosmological billiards was investigated. [207. The resulting dynamics was described in term of a quantized supersymmetric Bianchi type IX cosmology in 4D supergravity. Once again Kac-Moody structures were revealed.

A stable singularity formation result for expanding cosmological solutions to the Einstein-scalar field and Einstein-stiff fluid systems was proven in 208 for small perturbations of the spatially flat FLRW solution with topology $R \times T^{3}$. It was shown that the basic features of the perturbed solution in the collapsing direction closely resemble those of the FLRW solution, where its curvature blows up at a "big bang" singularity at $T^{3}$, confirming Penrose's strong cosmic censorship hypothesis for the "past-half" of near-FLRW solutions. the proof of linear stability for the Einstein-scalar field system can be generalized to control the non-linear terms. Recently a proof of stable curvature blow up (without any symmetry assumptions) towards the past singularity was given [209].

In 210] small perturbations of the family of FLRW cosmological background solutions to the Euler-Einstein system with a positive cosmological constant were studied. It was shown that the background solutions, which describe an initially uniform quiet fluid of positive energy density evolving in a de Sitter spacetime undergoing accelerated expansion with a linear equation of state, are globally future asymptotically stable under small irrotational perturbations. In particular, it was proven that the perturbed spacetimes, which have the topological structure $R \times T^{3}$, are future causally geodesically complete. 


\subsection{Spikes}

Spikes are a generic feature of solutions of partial differential equations. Therefore, it should be anticipated that spikes could be present in solutions of the field equations of GR. Indeed, in the oscillatory approach to an initial spacelike singularity, a spike could occur when a particular point in the state space becomes stuck in a previous Kasner epoch while nearby points begin to evolve to a subsequent Kasner epoch. In this way, it is plausible that spatial derivatives might have a significant effect at particular points in the early time cosmological dynamics. Since spikes can be arbitrarily narrow in the neighbourhood of the singularity, they are very difficult to simulate numerically. The mathematical analysis of spikes is also a challenge. It is an open problem to prove the existence of spikes within GR and to determine their dynamical effect on generic inhomogeneous spacelike singularities. Some mathematical justification for the existence of spikes is known [211. However, there has perhaps been greater success in obtaining exact solutions with spike-like behaviour [212.

Spikes were first observed in the numerical study of vacuum, orthogonally transitive, spatially inhomogeneous $G_{2}$ cosmologies [188, 213. Studies of $G_{2}$ and subsequently more general cosmological models therefore provide numerical evidence that the BKL dynamics generally occur except at very special isolated points (on one and two dimensional surfaces in three dimensional space) where spikes form 214. This then implies that the asymptotic locality part of the BKL conjecture is violated. Spikes also occur naturally in non-vacuum $G_{2}$ (and more general) spatially inhomogeneous cosmological models. Due to gravitational instability, the spikes then leave small imprints on the matter in the form of residual perturbations. To date the main focus has been on investigating spikes formed in the initial mixmaster regime and determining their imprint on matter and the subsequent effect on structure formation 215.

There are also non-local recurring spikes associated with cosmological singularities. However, it is believed that any spike formation occurs only for a set of time-lines of measure zero [13. There are many other outstanding questions regarding recurring spike behaviour and generic spacelike singularities [216]. In particular: How and where do spikes form? Do spikes experience interference or annihilation? Are there generic singularities that either do not have recurring spikes or have a dense set of recurring spikes? And are there solutions that undergo an infinite number of recurring spike transitions? In addition, to date the dynamical behaviour of solutions of the field equations in the asymptotic approach to a generic initial spacelike singularity for both vacuum and simple matter sources such as perfect fluids and scalar fields [187, 190] have been investigated. However, it is also of interest, of course, to study the structural stability of generic spacelike singularities in the presence of matter such as electromagnetic fields and form fields (in which the inhomogeneities may influence the cosmological singularity quite differently). 


\subsection{Numerical relativity and relativistic astrophysics}

Numerical analysis has always played an important role in any problem within GR that concerns non-linear phenomena. And recently there have been great advances in computational cosmology [217, 218]). Indeed, numerics have been used to support many of the conjectures presented here and, in turn, have led to significant theoretical progress and to the formulation of new problems in mathematical GR. We have discussed some of these problems earlier, including the important question of critical phenomena in gravitational collapse, which was originally discovered numerically [58].

Numerical analysis is also crucial for many problems in relativistic astrophysics. Indeed, recently progress in numerical computations has facilitated the investigation of the inspiral and violent merger of a pair of compact objects (e.g., black holes or neutron stars), in which an enormous quantity of gravitational waves is emitted. The determination of the predictions of emitted waveforms for binary systems for optimal detection and parameter extraction is a primary goal. Such an analysis proved very important in the recent LIGO observations [8]. More specifically, the analysis of the gravitational-wave signals resulting from black hole collisions give rise to very precise theoretical predictions which were then used as template waveforms and cross-correlated with the data obtained from the gravitational-wave detectors. In particular, analytic approximations (such as post Newtonian expansions, black hole perturbation theory and the effective one body approach) were used to model the orbital dynamics and gravitational-wave emission, and numerical relativity was then utilized to supplement these results at late times close to coalescence where such approximation schemes are no longer valid [7, 58.

To a large extent this problem has been solved in the case of a black-hole merger, although the relatively simple properties of the two-body non-linear gravity waveforms [119 have not been fully understood mathematically (and may involve new physics such as, for example, gravitational turbulence [219] and is also related to the final state conjecture). The same is not true in the case of neutron stars. However, there the difficulty is not with GR but with the matter; the binary inspiral problem is a problem in mathematical physics (utilizing appropriate approximation methods and numerical techniques, and introducing the correct physics in the neutron star case). Obtaining a complete solution (spacetime) for the two-body problem for particles coming in from infinity in the past to a stationary object to the future, is extremely difficult and beyond current analysis. A number of open problems, particularly concerning

the physical nature of the recent merger events and the gravitational waves detected by the LIGO-Virgo collaboration, have been discussed in 220. 


\section{Acknowledgements}

I would like to thank Piotr Bizon, Mihales Dafermos, Luis Lehner and Frans Pretorius for detailed comments on an earlier version of the manuscript, and Eric Poisson, Claes Uggla and Clifford Will for useful remarks. Financial support was provided by NSERC of Canada.

\section{References}

[1] S-T. Yau, ed. "Seminar on differential geometry", Annals of Math Studies (Princeton University Press Princeton, New Jersey, 1982).

[2] R. Penrose, Riv. Nuovo Cimento 1252 (1969); R. Penrose, Gen. Rel. Grav. 341141 (2002); R.Penrose, in "Theoretical Principles in Astrophysics and Relativity", eds. N. R. Lebovitz, W. H. Reid and P. O. Vandervoort (Univ. of Chicago Press, 1978)).

[3] R. Penrose, Ann. N.Y. Acad. 224125 (1973).

[4] R. Bartnik, "Some open problems in mathematical relativity", Conference on Mathematical Relativity (Canberra, 1988), ed. R. Bartnik, Proc. Centre Math. Anal. Austral. Nat. Univ., 19, Austral. Nat. Univ., Canberra, 244268 (1989). https : //projecteuclid.org/download/pdf 1 /euclid.pcma/1416335857

[5] J.-H. Eschenburg, J. Diff. Geom. 27477 (1988); see also G.J. Galloway, J. Geom. Phys. 6127 (1989); R. P. A. C. Newman, J. Diff. Geom. 31163 (1990).

[6] G. J. Galloway, "Some rigidity results for spatially closed spacetimes", pp. 2134, Mathematics of gravitation, Part I (Polish Acad. Sci., Warsaw, 1997); J.-H. Eschenburg and G. J. Galloway, Comm. Math. Phys. 148209 (1992); G. J. Galloway and C. Vega, Ann. Henri Poincare 152241 (2014).

[7] A. Le Tiec, B. Whiting and E. Poisson (eds.), Focus issue: Approaches to the two-body problem; http://www.iopscience.org/0264-9381/page/Focus Issues

[8] B. P. Abbott et al. [LIGO/Virgo Collaboration], Phys. Rev. Lett. 116 061102 (2016) arXiv:1602.03837.

[9] P. T. Chrusciel, G. J. Galloway and D. Pollack, "Mathematical general relativity: A sampler", Bull. Amer. Math. Soc. 47567 (2010) arXiv:1004.1016.

[10] S. Aretakis and I Rodnianski, "The Cauchy problem in general relativity", General relativity and gravitation (452479, Cambridge Univ. Press, Cambridge, 2015): https://mathscinet.ams.org/mathscinetgetitem? $\mathrm{mr}=3644042$. 
[11] A. Coley, "Open problems in mathematical physics", Phys. Scr. 9201 (2017).

[12] A. Rendall, Living Rev. Rel. 56 (2002) [https://arxiv.org/abs/grqc/0203012].

[13] L. Andersson, "The global existence problem in general relativity, The Einstein field equations and the large scale behaviour of gravitational fields", pp. 71-120 (Birkhuser, Basel, 2004) [arxiv/gr-qc/9911032].

[14] A. D. Ionescu and B. Pausader, "On the global regularity for a WaveKlein-Gordon coupled system" arXiv:1703.02846; P. G. LeFloch and Y. Ma, Comm. Math. Phys. 346603 (2016).

[15] M. Narita, Class. Quant. Grav. 196279 (2002) arXiv:gr-qc/0210088.

[16] Y. Choquet-Bruhat and R. Geroch, Comm. Math. Phys. 14329 (1969).

[17] Y. Choquet-Bruhat and J. York, "The Cauchy problem", in "General relativity and gravitation", vol. 1 99, ed. A. Held (Plenum, New York, 1980).

[18] H. Friedrich, Comm. Math. Phys. 107587 (1986).

[19] S. Dain and H. Friedrich, Comm. Math. Phys. 222569 (2001).

[20] J. A. Valiente Kroon, Comm. Math. Phys. 244133 (2004).

[21] H. Ringstrom, Class. Quant. Grav. 32124003 (2015) \& "Linear systems of wave equations on cosmological backgrounds with convergent asymptotics" arXiv:1707.02803.

[22] H. Ringstrom, "On proving future stability of cosmological solutions with accelerated expansion" in "Surveys in differential geometry 2015: One hundred years of general relativity", Surv. Differ. Geom. 20249 (Int. Press, Boston, MA, 2015).

[23] D. Christodoulou, and S. Klainerman, Commun. Pure Appl. Math. 43137 (1990).

[24] L. Andersson and V. Moncrief, "Future complete vacuum spacetimes", in "The Einstein equations and the large scale behaviour of gravitational fields", pp. 71-120 (Birkhuser, Basel, 2004) gr-qc/0303045].

[25] J. Isenberg, J. Math. Phys. 261024 (1985); V. Moncrief and J. Isenberg, Comm. Math. Phys. 89387 (1983).

[26] P. T. Chrusciel and A. D. Rendall, Ann. Physics 242349 (1995).

[27] V. Moncrief, Ann. Phys. 13287 (1981).

[28] P. T. Chrusciel, Ann. Phys. 202100 (1990). 
[29] J. Isenberg and V. Moncrief, Comm. Math. Phys. 86485 (1982).

[30] P. T. Chrusciel, J. Isenberg and V. Moncrief, Class. Quant. Grav. 71671 (1990).

[31] S. Kichenassamy and A. D. Rendall, Class. Quant. Grav. 151339 (1998).

[32] P. T. Chrusciel, "On uniqueness in the large of solutions of Einsteins equations; Mathematical aspects of classical field theory" pp. 235273 (Amer. Math. Soc., Providence, RI; Seattle, WA, 1991).

[33] P. T. Chrusciel and J. Isenberg, Phys. Rev. D 481616 (1993).

[34] Y. Choquet-Bruhat, Ann. Henri Poincare 21007 (2001).

[35] Y. Choquet-Bruhat, "Future complete Einsteinian space times with U(1) symmetry, the unpolarized case", The Einstein equations and the large scale behaviour of gravitational fields, pp71-120, ed. L. Andersson (Birkhuser, Basel, 2004) [ [gr-qc/0305060].

[36] P. T. Chrusciel and G. J. Galloway, Comm. Math. Phys. 193449 (1998).

[37] M. Kunzinger, R. Steinbauer, and M. Stojkovic, Differential Geom. Appl. 3414 (2014) \& Class. Quant. Grav. 32155010 (2015) arXiv:1502.00287; M. Kunzinger, R. Steinbauer, M. Stojkovic and J. A. Vickers, Class. Quant. Grav. 32075012 (2015) [arXiv:gr-qc/1411.4689].

[38] T. J. R. Hughes, T. Kato and J. E. Marsden, Arch. Rational Mech. Anal. 63273 (1976).

[39] S. Klainerman and I. Rodnianski, "The causal structure of microlocalized Einstein metrics" (2001) math.AP/0109174; S. Klainerman and I. Rodnianski, J. Hyperbolic Differ. Eqn. 185 (2004).

[40] D. Tataru, "Nonlinear wave equations", Proceedings of the ICM, Beijing 3 (2003) math.AP/0304397.

[41] J. M. M. Senovilla and D. Garfinkle, Class. Quant. Grav. 32124008 (2015) arXiv:1410.5226.

[42] R. Penrose, Phys. Rev. Lett. 1457 (1965).

[43] R. Penrose, "Singularities and time asymmetry", in "General Relativity: an Einstein Centenary Survey", eds. S. W. Hawking and W. Israel (Cambridge University Press, Cambridge, 1979).

[44] S. W. Hawking, Proc. Roy. Soc. London A294 511 (1966); ibid., A295 490 (1966); ibid., A300 187 (1967).

[45] R. Penrose and S. W. Hawking, Proc. Roy. Soc. Lond. A 314529 (1970). 
[46] S. W. Hawking and G. F. R. Ellis , "The large scale structure of spacetime" (Cambridge Univ. Press, Cambridge, 1973).

[47] J. M. M. Senovilla, "Singularity theorems in general relativity: achievements and open questions", Chapter 15 of Einstein and the Changing Worldviews of Physics, eds. C. Lehner, J. Renn and M. Schemmel, Einstein Studies 12 (Birkhauser, 2012)

[48] D. Christodoulou, "The formation of black holes in general relativity" (Monographs in Mathematics, European Mathematical Soc. Publishing House, Helsinki, 2009).

[49] S. Klainerman, J. Luk and I. Rodnianski, Invent. Math. 1981 (2014).

[50] S. Klainerman and I. Rodnianski, Acta Math. 208211 (2012); J. Luk and I. Rodnianski, "Nonlinear interactions of impulsive gravitational waves for the vacuum Einstein equations", Cambridge J. Math. arXiv:1301.1072; M. Dafermos, Astrisque 123352 (2013).

[51] X. An and J. Luk, "Trapped surfaces in vacuum arising dynamically from mild incoming radiation" arXiv:1409.6270; X. An, "Emergence of Apparent Horizon in Gravitational Collapse" [arXiv:1703.00118.

[52] L. Andersson and J. Metzger, Comm. Math. Phys., 290941 (2009); M. Eichmair, J. Diff. Geom. 83551 (2009); see also S. Alexakis, Class. Quant. Grav. 33115019 (2016) arXiv:1506.06400; L. Andersson, M. Eichmair and J. Metzger, "Jangs equation and its applications to marginally trapped surfaces" [arXiv: 1006.4601].

[53] A. J. S. Hamilton and P. P. Avelino, Phys. Rept. 4951 (2010) arXiv:0811.1926 gr-qc]; A. J. S. Hamilton, "Inflation followed by BKL collapse inside accreting, rotating black holes" arXiv:1703.01921 gr-qc]

[54] C. Misner, "Taub-NUT space as a counter-example to almost anything", in Relativity Theory and Astrophysics, ed. J. Ehlers (Amer. Math. Society, 1967).

[55] S. M. C. V. Goncalves, Phys. Rev. D 63064017 (2001) arXiv:gr-qc/0012032.

[56] D. Christodoulou, Ann. Math. 140607 (1994).

[57] D. Christodoulou, Ann. Math. 149183 (1999).

[58] M. W. Choptuik, L. Lehner and F. Pretorius, "Probing Strong Field Gravity Through Numerical Simulations", in "General Relativity and Gravitation: A Centennial Perspective", eds. A. Ashtekar, B. Berger, J. Isenberg and M. A. H. MacCallum (Cambridge University Press, 2015) arXiv:1502.06853. 
[59] D. Christodoulou, Class. Quant. Grav. 16 A23 (1999).

[60] M. Dafermos and J. Luk, "The interior of dynamical vacuum black holes I: the stability of the Kerr Cauchy horizon" [arxiv/1710.01722].

[61] H. Ringstrom, Living Rev. Rel. 132 (2010).

[62] R. M. Wald, "Gravitational collapse and cosmic censorship", in "Black Holes, Gravitational Radiation and the Universe", ed. by B.R. Iyer and B. Bhawal (Springer, Berlin, 1998) [arXiv: grqc/9710068].

[63] D. Christodoulou, Comm. Math. Phys. 105337 (1986) \& Comm. Math. Phys. 109613 (1987)

[64] J. Isenberg, "Progress on strong cosmic censorship, Mathematical aspects of classical field theory" pp. 403418 (Amer. Math. Soc., Providence, RI; Seattle, WA, 1992).

[65] O. J. C. Dias, J. E. Santos and B. Way, JHEP 12171 (2015).

[66] G. T. Horowitz, J. E. Santos and B. Way, Class. Quant. Grav. 33195007 (2016).

[67] P. Bizon and A. Rostworowski, Phys. Rev. Lett. 107031102 (2011).

[68] R. Schoen and S. T. Yau, Comm. Math. Phys. 6545 (1979) \& Comm. Math. Phys. 79231 (1981).

[69] V. Moncrief and D. M. Eardley, Gen. Rel. Grav. 13887 (1981).

[70] G. Huisken and T. Ilmanen, Math. Res. Notices 201045 (1997) \& J. Diff. Geom. 59353 (2001).

[71] A. D. Rendall, Helv. Phys. Acta 69490 (1996) gr-qc/9606049.

[72] G. Huisken, "An isoperimetric concept for mass and quasilocal mass", (Oberwolfach Rep., no. 2, 87, 2006); H. L. Bray, J. Diff. Geom. 59177 (2000).

[73] H. L. Bray and H. P. Roesch, "Null Geometry and the Penrose Conjecture" arXiv:1708.00941; ; H. Roesch, "Proof of a null penrose conjecture using a new quasi-local mass" arXiv:1609.02875; M. Mars and A. Soria, Class. Quant. Grav. 33115019 (2016).

[74] S. Dain, Gen. Rel. Grav 461 (2014) arXiv:1401.8166 \& Class. Quant. Grav. 29073001 (2012); M. Mars and A. Soria, Class. Quant. Grav. 33 115019 (2016) arXiv:1511.06242/gr-qc].

[75] P. Tod, Proc. Roy. Soc. Lond. A 388457 (1983). 
[76] G. Gibbons, "The isoperimetric and Bogomolny inequalities for black holes", in "Global Riemannian Geometry", ed. T. Willmore and N. Hitchin (Ellis Harwood Ltd., Chichester, 1984).

[77] K. S. Thorne, "Nonspherical Gravitational Collapse - A Short Review", in "Magic without Magic: John Archibald Wheeler", p. 231 ed. J. Klauder (Freeman, S. Francisco, 1972).

[78] J. M. M. Senovilla, Europhys. Lett. 8120004 (2008).

[79] M. W. Choptuik, Phys. Rev. Lett. 709 (1993).

[80] M. Reiterer and E. Trubowitz, "Choptuik's critical spacetime exists" arXiv:1203.3766.

[81] R. Bartnik and J. Mckinnon, Phys. Rev. Lett. 61141 (1988).

[82] D. Eardley and V. Moncrief, Comm. Math. Phys. 83171 (1982) \& Comm. Math. Phys. 83193 (1982).

[83] S. Klainerman and M. Machedon, Ann. Math. 14239 (1995).

[84] P. T. Chrusciel and J. Shatah, Asian J. Math. 1530 (1997).

[85] H. Friedrich, J. Diff. Geom. 34275 (1991).

[86] R. A. Bartnik, M. Fisher and T. A. Olinyk, J. Math. Phys. 51032504 (2010) arXiv:0907.3975.

[87] P. Bizon, Comm. Math. Phys. 21545 (2000); P. Bizon, T. Chmaj and Z. Tabor, Nonlinearity 141041 (2001).

[88] L. Andersson, N. Gudapati and J. Szeftel, "Global Regularity for the $2+1$ Dimensional Equivariant Einstein-Wave Map System" arXiv:1501.00616.

[89] J. Sterbenz and D. Tataru, Comm. Math. Phys. 298231 (2009) arXiv:0907.3148; J. Krieger and W. Schlag, "Large global solutions for energy supercritical nonlinear wave equations on $R^{3+1}$ " arXiv:1403.2913.

[90] P. Bizon and P. Biernat, Comm. Math. Phys. s00220-015-2404-y (2015); see also P. Bizon, Acta Physica Polonica B 331893 (2002).

[91] R. Donninger, Commun. Pure Appl. Math. 641095 (2011) \& Math Z. 278 1005 (2014).

[92] M. Choptuik, T. Chmaj and P. Bizon, Phys. Rev. Lett. 77424 (1996) arXiv:gr-qc/9603051.

[93] H. Andreasson, Living Rev. Rel. 144 (2011) arXiv:1106.1367.

[94] D. Christodoulou and S. Klainerman, "The global nonlinear stability of the Minkowski space" (Princeton mathematical series, 41, Princeton University Press, 1993). 
[95] H. Lindblad and I. Rodnianski, Comm. Math. Phys. 25643 (2005); L. Bieri and N. Zipser, "Extensions of the stability theorem of the Minkowski space in general relativity", AMS/IP Studies in Advanced Mathematics, 45 (American Mathematical Society, Providence, RI; International Press, Cambridge, MA, 2009).

[96] D. Fajman, J. Joudioux and J. Smulevici, "The Stability of the Minkowski space for the Einstein-Vlasov system" arXiv:1707.06141; H. Lindblad and M. Taylor, Commun. Part. Diff. Eq. arXiv:1707.06079; M. Taylor, Ann. PDE 39 (2017) arXiv:1602.02611.

[97] G. Bunting and A. K. M. Masood-ul-Alam, Gen. Rel. Grav. 19147 (1987).

[98] W. Israel, Comm. Math. Phys. 8245 (1968).

[99] R. P. Kerr, Phys. Rev. Lett. 11237 (1963).

[100] B. Carter, in Black Holes, 1972 Les Houches Lectures, eds. B. DeWitt and C. DeWitt (Gordon and Breach, NY, 1973); B. Carter, Comm. Math. Phys. 99563 (1985).

[101] D. C. Robinson, Phys. Rev. Lett. 34905 (1975).

[102] E. Newman, J. Math. Phys. 6918 (1965).

[103] P. Mazur, J. Phys. A 153173 (1982).

[104] M. Heusler, Living Rev. Rel. 1 6 (1998); http://www.livingreviews.org/Articles/Volume1/1998-6heusler.

[105] S. Chandrasekhar, "Mathematical Theory of Black Holes" (Oxford University Press, 1983).

[106] G. Holzegel, Class. Quant. Grav. 33205001 (2016).

[107] M. Dafermos, G. Holzegel and I. Rodnianski, "The linear stability of the Schwarzschild solution to gravitational perturbations", 146 pages (2016) arXiv:1601.06467.

[108] S. Klainerman and J. Szeftel, "Global Nonlinear Stability of Schwarzschild Spacetime under Polarized Perturbations", 425 pages arXiv:1711.07597.

[109] M. Dafermos, I. Rodnianski and Y. Shlapentokh-Rothman, "Decay for solutions of the wave equation on Kerr exterior spacetimes: the full subextremal case" arXiv:1402.7034.

[110] M. Dafermos and I. Rodnianski, "Lectures on black holes and linear waves", Clay Mathematics Proceedings 1797 (2008) arXiv:0811.0354].

[111] M. Dafermos, G. Holzegel and I. Rodnianski, "Boundedness and decay for the Teukolsky equation on Kerr spacetimes I" [arxiv/1711.07944]. 
[112] M. Zilhao, V. Cardoso, C. Herdeiro, L. Lehner and U. Sperhake, Phys. Rev. D 90124088 (2014) arXiv:1410.0694.

[113] J. L. Friedman, Comm. Math. Phys. 63243 (1978) [arxiv/1608.02035].

[114] G. Moschidis, "A proof of Friedman's ergosphere instability for scalar waves" [arxiv/1608.02035].

[115] S. Aretakis, "Horizon Instability of Extremal Black Holes" [arxiv/1206.6598].

[116] P. Hintz and A. Vasy, "The global non-linear stability of the Kerr-de Sitter family of black holes" arXiv:1606.04014; V. Schlue, Comm. Math. Phys. 334977 (2015).

[117] G. Holzegel and J. Smulevici, Anal. PDE 71057 (2014) arXiv:1303.5944.

[118] M. Dafermos, G. Holzegel and I. Rodnianski, "A scattering theory construction of dynamical vacuum black holes", to appear in J. Diff. Geom. (2013) arXiv:1306.5364.

[119] H. Yang, V. Paschalidis, K. Yagi, L. Lehner, F. Pretorius and N. Yunes, Phys. Rev. D 97024049 (2018) arXiv:1707.00207.

[120] J. Ehlers, "The Newtonian limit of general relativity", in "Classical mechanics and relativity: relationship and consistency", ed. G. Ferrarese, (Bibliopolis, Naples, 1991); see also J. Ehlers and T. Buchert, Gen. Rel. Grav. 29733 (1997); T. A. Oliynyk, Comm. Math. Phys. 276131 (2007) arXiv:astro-ph/9510056.

[121] J. Ehlers, "Folklore in relativity and what is really known", in General Relativity and Gravitation" 11, ed. M.A. H. MacCallum (Springer, Stockholm 1986).

[122] R. Bartnik, Comm. Math. Phys. 117615 (1988); P. T. Chrusciel, J. Isenberg and D. Pollack, Comm. Math. Phys. 25729 (2005).

[123] J. Dilts and M. Holst, "When Do Spacetimes Have Constant Mean Curvature Slices?" arXiv:1710.03209.

[124] A. Coley, S. Hervik and N. Pelavas, Class. Quant. Grav. 26025013 (2009) arXiv:0904.4877.

[125] A. Coley, S. Hervik and N. Pelavas, Class. Quant. Grav. 27102001 (2010) [arXiv1003.2373]; see also A. Coley and S. Hervik, Gen. Rel. Grav. 432199 (2011).

[126] H. Stephani, D. Kramer, M. MacCallum, C. Hoenselaers and E. Herlt, "Exact Solutions of Einsteins Field Equations" (Cambridge University Press, Cambridge, second ed., 2003). 
[127] A. Coley, D. D. McNutt, and A. Shoom, Phys. Lett. B 771131 (2017); A. Coley and D. D. McNutt, Class. Quant. Grav. 35025013 (2018) arXiv:1710.08773.

[128] L. Bieri, D. Garfinkle and N. Yunes, AMS Notices 6407 (2017) arXiv:1710.03272 ; L. Bieri, D. Garfinkle and S.-T. Yau, in "The Centenary of General Relativity", volume 20 of "Surveys in Differential Geometry" arXiv:1505.05213.

[129] D. Christodoulou, Phys. Rev. Letts. 671486 (1991).

[130] L. Bieri, D. Garfinkle and N. Yunes, "Gravitational wave memory in de Sitter spacetime" arXiv:1706.02009; L. Bieri, D. Garfinkle and S.-T. Yau, Phys. Rev. D 94064040 (2016) arXiv:1509.01296.

[131] M. Ishak, "Testing general relativity in cosmology" arXiv:1806.10122.

[132] S. W. Goode and J. Wainwright, Class. Quant. Grav. 299 (1985); S. W. Goode, A. A. Coley and J. Wainwright, Class. Quant. Grav. 9445 (1992) arXiv:0810.3744; C C. M. Claudel and K. P. Newman, Proc. R. Soc. London, Ser. A 4543 (1998).

[133] J, Middleton and J. D. Barrow, Phys. Rev. D 7710352 (2008) arXiv:0801.4090; I. V. Kirnos, A. N. Makarenko, S. A. Pavluchenko and A. V. Toporensky, Gen. Rel. Grav. 422633 (2010) [arXiv:gr-qc/0906.0140]; J. D. Barrow and S. Hervik, Phys. Rev. D 81023513 (2010) arXiv:0911.3805.

[134] S. W. Hawking, Nature 248, 30 (1974); S. W. Hawking, Phys. Rev. D14, 2460 (1976); S. Hawking, Comm. Math. Phys. 43199 (1975).

[135] C Barcelo, R Carballo-Rubioy and L. J. Garayz, JHEP 05054 (2017) arXiv:1701.09132.

[136] J. M. Maldacena, Int. J. Theor. Phys. 381113 (1999); J. M. Maldacena, Adv. Theor. Math. Phys. 2231 (1998).

[137] I. Klebanov and J. Maldacena, Physics Today 6228 (2009).

[138] G. Holzegel and A. Shao, "Unique continuation from infinity in asympotically Anti-de Sitter spacetimes II: Non-static boundaries" arXiv:1608.07521/gr-qc].

[139] H. Friedrich, J. Geom. Phys. 17125 (1995)

[140] P. Bizon, Gen. Rel. Grav. 461724 (2014) arXiv:1312.5544.

[141] P. Bizon, M. Maliborski and A. Rostworowski, Phys. Rev. Lett. 115 081103 (2015).

[142] M. Dafermos and G. Holzegel 2006 Seminar at DAMTP (University of Cambridge) available at: https://dpmms.cam.ac.uk/ md384/ADSinstability.pdf 
[143] M. T. Anderson, Class. Quant. Grav. 236935 (2006).

[144] M. Maliborski and A. Rostworowski, Phys. Rev. Lett. 111051102 (2013) arXiv:1303.3186.

[145] O. J. C. Dias, G. T. Horowitz and J. E. Santos, Class. Quant. Grav. 29194002 (2012) arXiv:1109.1825; O. J. C. Dias, and J. E. Santos, "AdS nonlinear instability: breaking spherical and axial symmetries" arXiv:1705.03065; A. Rostworowski, Class. Quant. Grav. 33 23LT01 (2016)] arXiv:1612.00042.

[146] O. J. C. Dias, G. T. Horowitz, D. Marolf and J. E. Santos, Class. Quant. Grav. 29235019 (2012); S. R. Green, A. Maillard, L. Lehner and S. L. Liebling, Phys. Rev. D 92084001 (2015) arXiv:1507.08261.

[147] G. Martinon, "The instability of anti-de Sitter space-time" arXiv:1708.05600].

[148] G. Moschidis, "The Einstein null dust system in spherical symmetry with an inner mirror: structure of the maximal development and Cauchy stability" arXiv:1704.08685] \& "A proof of the instability of AdS for the Einstein null dust system with an inner mirror" arXiv:1704.08681].

[149] J. Jalmuzna, A. Rostworowski and P. Bizon, Phys. Rev. D 84085021 (2011).

[150] M Green, J Schwarz and E Witten, "Superstring Theory" (Cambridge: Cambridge University Press, 1988); J. Polchinski, "String Theory" (Cambridge: Cambridge University Press 2005)

[151] R. Emparan and H. S. Reall, Living Rev. Rel. 116 (2008) arXiv:0801.3471.

[152] A. Coley, R. Milson, V. Pravda and A. Pravdova, Class. Quant. Grav. 21 L35 (2004) gr-qc/0401008; A. Coley, Class. Quant. Grav. 25033001 (2008) arXiv:0710.1598.

[153] G. J. Galloway and J. M. M. Senovilla, Class. Quant. Grav. 27152002 (2010).

[154] R. Schoen and S.-T. Yau. "Positive Scalar Curvature and Minimal Hypersurface Singularities" arXiv:1704.05490.

[155] L. Lehner and F. Pretorius, Phys. Rev. Lett. 105101102 (2010).

[156] R. Gregory and R. Laflamme, Phys. Rev. Lett. 702837 (1993).

[157] R. Emparan and H. S. Reall, Phys. Rev. Lett. 88101101 (2002).

[158] J. E. Santos and B. Way, Phys. Rev. Lett. 114, 221101 (2015); K. Tanabe, JHEP 02151 (2016); P. Figueras, M. Kunesch, and S. Tunyasuvunakool, Phys. Rev. Lett. 116071102 (2016). 
[159] R. C. Myers and M. J. Perry, Ann. Phys. 172304 (1986).

[160] R. Emparan and R. C. Myers, JHEP 09025 (2003).

[161] O. J. C. Dias, P. Figueras, R. Monteiro, J. E. Santos, and R. Emparan, Phys. Rev. D 80111701 (2009); P. Figueras, M. Kunesch, L. Lehner, and S. Tunyasuvunakool, Phys. Rev. Letts. 118151103 (2017).

[162] M. Henneaux, Khalatnikov-Lifshitz analysis in Quantum Mechanics of Fundamental Systems: the Quest for Beauty and Simplicity - Claudio Bunster Festsschrift arXiv:0806.4670].

[163] W. E. East, M. Kleban, A. Linde and L. Senatore, JCAP 09010 (2016) arXiv:1511.05143; J. Braden, M. C. Johnson, H. V. Peiris and A. Aguirre, Phys. Rev. D 96023541 (2017) arXiv:1604.04001.

[164] R. Brandenberger and P. Peter, Found. Phys. $47 \quad 797$ (2017) arXiv:1603.05834 hep-th].

[165] D. Garfinkle, W. C. Lim, F. Pretorius and P. J. Steinhardt, Phys. Rev. D 78083537 (2008); B. Xue, D. Garfinkle, F. Pretorius and P. J. Steinhardt, Phys. Rev. D 88083509 (2013).

[166] R. E. Allen and S. Lidstrom, "Life, the universe, and everything: 42 fundamental questions", Phys. Scr. 92012501 (2017) [Focus Issue on 21st Century Frontiers].

[167] K. Freese, "Status of Dark Matter in the Universe" arXiv:1701.01840.

[168] E. Witten, "The cosmological constant from the viewpoint of string theory", in Sources and Detection of Dark Matter and Dark Energy in the Universe, ed. D. B. Cline pages 27-36 (Springer, Berlin, Heidelberg, 2001).

[169] P. Steinhardt and N. Turok, Science $\mathbf{3 1 2} 1180 \quad$ (2006) arXiv:astro-ph/0605173.

[170] S. Weinberg, Rev. Mod. Phys. 611 (1989).

[171] A. Padilla, "Lectures on the Cosmological Constant Problem" arXiv:1502.05296.

[172] S. Weinberg, Phys. Rev. Lett. 592607 (1987).

[173] A. G. Riess et al., Astron. J. 1161009 (1998); S. Perlmutter et al., Astrophys. J. 517565 (1999).

[174] T. Buchert, A. A. Coley, H. Kleinert, B. F. Roukema and D. L. Wiltshire, Int. J. Mod. Phys. D 251630007 (2016) arXiv:1512.03313. 
[175] J. Einasto, "Yakov Zeldovich and the Cosmic Web Paradigm", in Proc. IAU Symp. 308, eds. R. van de Weygaert, S. Shandarin, E. Saar, J. Einasto (Cambridge Univ. Press, 2017) arXiv:1410.6932; F. Hoyle and M. S. Vogeley, Astrophys. J. 566641 (2002) [arXiv:astro-ph/0109357]; Astrophys. J. 607751 (2004) arXiv:astro-ph/0312533; D. C. Pan, M. S. Vogeley, F. Hoyle, Y. Y. Choi, and C. Park, Mon. Not. R. Astron. Soc. 421926 (2012) arXiv:1103.4156.

[176] J. Brannlund, R. van den Hoogen and A. Coley, "Averaging geometrical objects on a differentiable manifold" arXiv:1003.2014; R. van den Hoogen, J. Math. Phys. 58122501 (2017); A. A. Coley, Class. Quant. Grav. 27 245017 (2010) arXiv:0908.4281]; see also T. Buchert et al., Class. Quant. Grav. 32215021 (2015) arXiv:1505.07800.

[177] R. M. Zalaletdinov, Gen. Rel. Grav. 241015 (1992) \& Gen. Rel. Grav. 25673 (1993) arXiv:gr-qc/9703016; M. Mars and R. M. Zalaletdinov, J. Math. Phys. 384741 (1997); A. A. Coley, N. Pelavas and R. M. Zalaletdinov, Phys. Rev. Letts. 95151102 (2005) arXiv:gr-qc/0504115.

[178] L. Andersson, "Cosmological Models and Stability", in "General Relativity, Cosmology and Astrophysics, Fundamental Theories of Physics", 177 p. 277 (Springer International Publishing Switzerland, 2014; ISBN 978-3319-06348-5).

[179] J. D. Barrow, G. J. Galloway and F. J. Tipler, Mon. Not. R. Astron. Soc. 223835 (1986).

[180] X. Lin and R. M. Wald, Phys. Rev. D 403280 (1989) \& 412444 (1990).

[181] H. Friedrich, J. Geom. Phys. 3101 (1986).

[182] R. Wald, Phys. Rev. D 282118 (1983).

[183] A. D. Rendall, Math. Proc. Camb. Phil. Soc. 118511 (1995).

[184] A. A. Coley, "Dynamical systems and cosmology" (Kluwer Academic, Dordrecht: ISBN 1-4020-1403-1, 2003).

[185] J. M. Heinzle and A. D. Rendall, Comm. Math. Phys. 2691 (2007); H. Ringstrom, Comm. Math. Phys. 290155 (2009).

[186] L. G. Jensen and J. A. Stein-Schabes, Phys. Rev. D 351146 (1987).

[187] E. M. Lifshitz and I. M. Khalatnikov, Adv. Phys. 12185 (1963); V. A. Belinskii, I. M. Khalatnikov, and E. M. Lifschitz, Adv. Phys. 19, 525 (1970); ibid. 31639 (1982); V. A. Belinskii and I. M. Khalatnikov, Soviet Scientific Review Section A: Physics Reviews 3555 (1981).

[188] B. K. Berger and V. Moncrief, Phys. Rev. D 484676 (1993); B. K. Berger, Living Rev. Rel. 51 (2002). 
[189] D. Garfinkle, Phys. Rev. Lett. 93161101 (2004); D. Garfinkle, Class. Quant. Grav. 24 S295 (2007).

[190] J. Wainwright and G. F. R. Ellis, "Dynamical systems in cosmology" (Cambridge University Press, Cambridge, 1997).

[191] C. Uggla, H. van Elst, J. Wainwright and G. F. R. Ellis, Phys. Rev. D 68 103502 (2003).

[192] J. M. Heinzle and C. Uggla, Class. Quant. Grav. 26075016 (2009) arXiv:0901.0776.

[193] A. D. Rendall, Class. Quant. Grav. 142341 (1997).

[194] H. Ringstrom, Class. Quant. Grav. 17713 (2000): H. Ringstrom, Annales Henri Poincare 2405 (2001).

[195] B. Brehm, "Bianchi VIII and IX vacuum cosmologies: Almost every solution forms particle horizons and converges to the Mixmaster attractor" (Doctoral Thesis, Freie Universitat Berlin, 2016) [arXiv:1606.08058, 2016].

[196] J. M. Heinzle, C. Uggla and N. Rohr, Adv. Theor. Math. Phys. 13293 (2009).

[197] V. A. Belinskii, L. P. Grishchuk, Ya. B. Zeldovich and I. M. Khalatnikov, Sov. Phys. JETP 62195 (1986).

[198] C. G. Hewitt, J. T. Horwood and J. Wainwright, Class. Quant. Grav. 20 1743 (2003).

[199] V. G. LeBlanc, Class. Quant. Grav. 142281 (1997); V. G. LeBlanc, D. Kerr, and J. Wainwright, Class. Quant. Grav. 12513 (1995).

[200] C. G. Hewitt, R. Bridson and J. Wainwright, Gen. Rel. Grav. 3365 (2001).

[201] S. Hervik, R. J. van den Hoogen, W. C. Lim and A. A. Coley, Class. Quant. Grav. 243859 (2007) arXiv:gr-qc/0703038.

[202] C. Uggla, Int. J. Mod. Phys. D 221330002 (2013) arXiv:1306.6527 \& Gen. Rel. Grav. 451669 (2013) arXiv:1304.6905.

[203] R. Maier, I. Damiao Soares and E. V. Tonini, Class. Quant. Grav. 32 235001 (2015) arXiv:1505.06189.

[204] R. Temam, "Infinite-Dimensional Dynamical Systems in Mechanics and Physics" (Springer-Verlag, 1988); R. Temam, "Dynamical systems in infinite dimensions", in "The Connection between Infinite-Dimensional and Finite-Dimensional Dynamical Systems", eds. B. Nicolaenko, C. Foias and R. Temam (American Mathematical Society, 1988). 
[205] T. Damour, M. Henneaux and H. Nicolai, Class. Quant. Grav. 20 R145 (2003) hep-th/0212256; T. Damour, M. Henneaux and H. Nicolai, Phys. Rev. Lett. 89221601 (2002) hep-th/0207267]; see also T. Damour and H. Nicolai, Class. Quant. Grav. 222849 (2005).

[206] T. Damour and C. Hillmann, JHEP 0908100 (2009) arXiv:0906.3116 [hep-th]]; A. Kleinschmidt, M. Koehn and H. Nicolai, Phys. Rev. D 80 061701 (2009) [arXiv:0907.3048 [gr-qc]].

[207] T. Damour and P. Spindel, Class. Quant. Grav. 30162001 (2013) arXiv:1304.6381[gr-qc]] \& Phys. Rev. D 9103509 (2014) arXiv:1406.1309 [gr-qc]] \& Phys. Rev. D 95126011 (2017) arXiv:1704.08116.

[208] I. Rodnianski and J. Speck, "Stable Big Bang Formation in Near-FLRW Solutions to the Einstein-Scalar Field and Einstein-Stiff Fluid Systems" [arxiv.org/1407.6298].

[209] I. Rodnianski and J. Speck, "On the nature of Hawking's incompleteness for the Einstein-vacuum equations" arXiv:1804.06825.

[210] I. Rodnianski and J. Speck, "The Stability of the Irrotational EulerEinstein System with a Positive Cosmological Constant" [arxiv/0911.5501].

[211] H. Ringstrom, Math. Proc. Camb. Phil. Soc. 136485 (2004); H. Ringstrom, Class. Quant. Grav. 21 S305 (2004).

[212] W. C. Lim, Class. Quant. Grav. 32162001 (2015) arXiv:1507.02754; A. A. Coley, D. Gregoris and W. C. Lim, Class. Quant. Grav. (2016) arXiv:1606.07177.

[213] W. C. Lim, L. Andersson, D. Garfinkle and F. Pretorius, Phys. Rev. D 79103526 (2009) arXiv:0904.1546.

[214] B. K. Berger, J. Isenberg and M. Weaver, Phys. Rev. D 64084006 (2001).

[215] A. A. Coley and W. C. Lim, Phys. Rev. Lett. 108191101 (2012) arXiv:1205.2142; W. C. Lim and A. A. Coley, Class. Quant. Grav. 31 015020 (2014) arXiv:1311.1857.

[216] J. M. Heinzle and C. Uggla, Gen. Rel. Grav. 45939 (2013) arXiv:1212.5500/gr-qc]; J. M. Heinzle, C. Uggla and W. C. Lim, Phys. Rev. D 86104049 (2012) arXiv:1206.0932/gr-qc].

[217] A. Coley, L. Lehner, F. Pretorius and D. Wiltshire, "Computational Issues in Mathematical Cosmology" (2017); http://cms.iopscience.iop.org/alfresco/d/d/workspace/SpacesStore/ 83f10d6e-0b33-11e7-9a47-19ee90157113/Overview-CC.pdf 
[218] E. Bentivegna and M. Bruni, Phys. Rev. Lett. 116251302 (2016) arXiv:1511.05124; E. Bentivegna, Phys. Rev. D 95044046 (2017) arXiv:1610.05198; J. T. Giblin, J. B. Mertens and G. D. Starkman, Phys. Rev. Lett. 116251301 (2016), ibid. Phys. Rev. D 93124059 (2016) arXiv:1511.01105, ibid. "A cosmologically motivated reference formulation of numerical relativity" arXiv:1704.04307; J. Adamek, D. Daverio, R. Durrer and M. Kunz, Nature Physics 12346 (2016) arXiv:1509.01699; J. Adamek, C. Clarkson, D. Daverio, R. Durrer and M. Kunz, "Safely smoothing spacetime: backreaction in relativistic cosmological simulations" arXiv:1706.09309; H. Macpherson, D. J. Price and P. D. Lasky, "Einstein's Universe: cosmological structure formation in numerical relativity" arXiv:1807.01711.

[219] J. R. Westernacher-Schneider, L. Lehner and Y. Oz, JHEP 12067 (2015) arXiv:1510.00736.

[220] L. Barack et al., "Black holes, gravitational waves and fundamental physics: a roadmap" arXiv:1806.05195. 